\title{
EFFECTIVE RESULTS FOR DIOPHANTINE EQUATIONS OVER FINITELY GENERATED DOMAINS
}

\author{
ATTILA BÉRCZES, JAN-HENDRIK EVERTSE, AND KÁLMÁN GYŐRY
}

\section{Introduction.}

Let $A$ be an arbitrary integral domain of characteristic 0 that is finitely generated over $\mathbb{Z}$. We consider Thue equations $F(x, y)=\delta$ in $x, y \in A$, where $F$ is a binary form with coefficients from $A$ and $\delta$ is a non-zero element from $A$, and hyper- and superelliptic equations $f(x)=\delta y^{m}$ in $x, y \in A$, where $f \in A[X], \delta \in A \backslash\{0\}$ and $m \in \mathbb{Z}_{\geq 2}$.

Under the necessary finiteness conditions we give effective upper bounds for the sizes (defined in Section 2) of the solutions of the equations in terms of appropriate representations for $A, \delta, F, f, m$. These results imply that the solutions of these equations can be determined in principle. Further, we consider the Schinzel-Tijdeman equation $f(x)=\delta y^{m}$ where $x, y \in A$ and $m \in \mathbb{Z}_{\geq 2}$ are the unknowns and give an effective upper bound for $m$.

We mention that results from the existing literature deal only with equations over restricted classes of finitely generated domains whereas we do not have to impose any restrictions on $A$. Further, our upper bounds for the sizes of the solutions $x, y$ and $m$ are new, also for the special cases considered earlier. Our proofs are a combination of existing effective results for Thue equations and hyper- and superelliptic equations over number fields and

2010 Mathematics Subject Classification: 11D41, 11D59, 11D61.

Keywords and Phrases: Thue equations, hyperelliptic equations, superelliptic equations, Schinzel-Tijdeman equation, effective results, Diophantine equations over finitely generated domains.

The research was supported in part by the Hungarian Academy of Sciences, and by grants K100339 (A.B., K.G.), NK104208 (A.B., K.G.) and K75566 (A.B.) of the Hungarian National Foundation for Scientific Research. The work is supported by the TÁMOP 4.2.1./B-09/1/KONV-2010-0007 project. The project is implemented through the New Hungary Development Plan, co-financed by the European Social Fund and the European Regional Development Fund. 
over function fields, and a recent effective specialization method of Evertse and Győry [9].

We give a brief overview of earlier results. A major breakthrough in the effective theory of Diophantine equations was established by A. Baker in the 1960 's. Using his own estimates for linear forms in logarithms of algebraic numbers, he obtained effective finiteness results, i.e., with explicit upper bounds for the absolute values of the solutions, for Thue equations [2] and hyper- and superelliptic equations $[3]$ over $\mathbb{Z}$. Schinzel and Tijdeman [17] were the first to consider superelliptic equations $f(x)=\delta y^{m}$ over $\mathbb{Z}$ where also the exponent $m$ was taken as an unknown and gave an effective upper bound for $m$. Their proof also depends on Baker's linear forms estimates.

The effective results of Baker and of Schinzel and Tijdeman were extended to equations where the solutions $x, y$ are taken from larger integral domains; we mention here Coates [8], Sprindžuk and Kotov [19] (Thue equations over $\mathcal{O}_{S}$, where $\mathcal{O}_{S}$ is the ring of $S$-integers of an algebraic number field), Trelina [21], Brindza [6] (hyper- and superelliptic equations over $\mathcal{O}_{S}$ ), Győry [11] (Thue equations over a restricted class of integral domains finitely generated over $\mathbb{Z}$ that contain transcendental elements), Brindza [7] and Végső [22] (hyper- and superelliptic equations and the Schinzel-Tijdeman equation over the class of domains considered by Györy). These last mentioned works of Győry, Brindza and Végső were based on an effective specialization method developed by Györy in the 1980's [11], [12].

Recently, Evertse and Győry [9] extended Győry's specialization method so that it can now be used to prove effective results for Diophantine equations over arbitrary finitely generated domains $A$ over $\mathbb{Z}$, without any further restriction on $A$ whatsoever. They applied this to unit equations $a x+b y=c$ in units $x, y$ of $A$, and gave an effective upper bound for the sizes of the solutions $x, y$ in terms of appropriate representations for $A, a, b, c$. In their method of proof, Evertse and Győry used existing effective results for $S$-unit equations over number fields and function fields, and combined these with their general specialization method.

The approach of Evertse and Györy can be applied to various other classes of Diophantine equations. In the present paper, we have worked out the consequences for Thue equations, hyper-and superelliptic equations, and Schinzel-Tijdeman equations. 


\section{Results}

We first introduce the necessary notation and then state our results.

2.1. Notation. Let $A=\mathbb{Z}\left[z_{1}, \ldots, z_{r}\right]$ be a finitely generated integral domain of characteristic 0 which is finitely generated over $\mathbb{Z}$. We assume that $r>0$. We have

$$
A \cong \mathbb{Z}\left[X_{1}, \ldots, X_{r}\right] / I
$$

where $I$ is the ideal of polynomials $f \in \mathbb{Z}\left[X_{1}, \ldots, X_{r}\right]$ such that $f\left(z_{1}, \ldots, z_{r}\right)=$ 0 . The ideal $I$ is finitely generated, say

$$
I=\left(f_{1}, \ldots, f_{t}\right) .
$$

We may view $f_{1}, \ldots, f_{t}$ as a representation for $A$. Recall that a necessary and sufficient condition for $A$ to be a domain of characteristic zero is that $I$ be a prime ideal with $I \cap \mathbb{Z}=(0)$. Given a set of generators $\left\{f_{1}, \ldots, f_{t}\right\}$ for $I$ this can be checked effectively (see for instance Aschenbrenner [1, Cor. 6.7, Lemma 6.1] but this follows already from work of Hermann [14]).

Denote by $K$ the quotient field of $A$. For $\alpha \in A$, we call $f$ a representative for $\alpha$, or we say that $f$ represents $\alpha$, if $f \in \mathbb{Z}\left[X_{1}, \ldots, X_{r}\right]$ and $\alpha=f\left(z_{1}, \ldots, z_{r}\right)$. Further, for $\alpha \in K$ we call $(f, g)$ a pair of representatives for $\alpha$, or say that $(f, g)$ represents $\alpha$ if $f, g \in \mathbb{Z}\left[X_{1}, \ldots, X_{r}\right], g \notin I$ and $\alpha=f\left(z_{1}, \ldots, z_{r}\right) / g\left(z_{1}, \ldots, z_{r}\right)$.

Using an ideal membership algorithm for $\mathbb{Z}\left[X_{1}, \ldots, X_{r}\right]$ (see e.g., Aschenbrenner [1, Theorem A] but such algorithms were probably known in the 1960's), one can decide effectively whether two polynomials $f^{\prime}, f^{\prime \prime} \in$ $\mathbb{Z}\left[X_{1}, \ldots, X_{r}\right]$ represent the same element of $A$, i.e., $f^{\prime}-f^{\prime \prime} \in I$, or whether two pairs of polynomials $\left(f^{\prime}, g^{\prime}\right),\left(f^{\prime \prime}, g^{\prime \prime}\right)$ in $\mathbb{Z}\left[X_{1}, \ldots, X_{r}\right]$ represent the same element of $K$, i.e., $g^{\prime} \notin I, g^{\prime \prime} \notin I$ and $f^{\prime} g^{\prime \prime}-f^{\prime \prime} g^{\prime} \in I$.

Given a non-zero polynomial $f \in \mathbb{Z}\left[X_{1}, \ldots, X_{r}\right]$, we denote by $\operatorname{deg} f$ its total degree and by $h(f)$ its logarithmic height, that is the logarithm of the maximum of the absolute values of its coefficients. Then the size of $f$ is defined by

$$
s(f):=\max (1, \operatorname{deg} f, h(f)) .
$$

Further, we define $s(0):=1$. It is clear that there are only finitely many polynomials in $\mathbb{Z}\left[X_{1}, \ldots, X_{r}\right]$ of size below a given bound, and these can be determined effectively. 
Throughout the paper we shall use the notation $O(\cdot)$ to denote a quantity which is $c$ times the expression between the parentheses, where $c$ is an effectively computable positive absolute constant which may be different at each occurrence of the $O$-symbol. Further, throughout the paper we write

$$
\log ^{*} a:=\max (1, \log a) \quad \text { for } a>0, \quad \log ^{*} 0:=1 .
$$

2.2. Thue equations. We consider the Thue equation over $A$,

$$
F(x, y)=\delta \quad \text { in } \quad x, y \in A,
$$

where

$$
F(X, Y)=a_{0} X^{n}+a_{1} X^{n-1} Y+\cdots+a_{n} Y^{n} \in A[X, Y]
$$

is a binary form of degree $n \geq 3$ with discriminant $D_{F} \neq 0$, and $\delta \in A \backslash\{0\}$. Choose representatives

$$
\tilde{a_{0}}, \tilde{a_{1}}, \ldots, \tilde{a_{n}}, \tilde{\delta} \in \mathbb{Z}\left[X_{1}, \ldots, X_{r}\right]
$$

of $a_{0}, a_{1}, \ldots, a_{n}, \delta$, respectively. To ensure that $\delta \neq 0$ and $D(F) \neq 0$, we have to choose the representatives in such a way that $\tilde{\delta} \notin I, D_{\tilde{F}} \notin I$ where $D_{\tilde{F}}$ is the discriminant of $\tilde{F}:=\sum_{j=0}^{n} \tilde{a_{j}} X^{n-j} Y^{j}$. These last two conditions can be checked by means of the ideal membership algorithm mentioned above. Let

$$
\left\{\begin{array}{l}
\max \left(\operatorname{deg} f_{1}, \ldots, \operatorname{deg} f_{t}, \operatorname{deg} \tilde{a_{0}}, \operatorname{deg} \tilde{a_{1}}, \ldots, \operatorname{deg} \tilde{a_{n}}, \operatorname{deg} \tilde{\delta}\right) \leq d \\
\max \left(h\left(f_{1}\right), \ldots, h\left(f_{t}\right), h\left(\tilde{a_{0}}\right), h\left(\tilde{a_{1}}\right), \ldots, h\left(\tilde{a_{n}}\right), h(\tilde{\delta})\right) \leq h
\end{array}\right.
$$

where $d \geq 1, h \geq 1$.

Theorem 2.1. Every solution $x, y$ of equation (2.1) has representatives $\tilde{x}, \tilde{y}$ such that

$$
s(\tilde{x}), s(\tilde{y}) \leq \exp \left(n !(n d)^{\exp O(r)}(h+1)\right) .
$$

The exponential dependence of the upper bound on $n !, d$ and $h+1$ is coming from a Baker-type effective result for Thue equations over number fields that is used in the proof. The bad dependence on $r$ is coming from the effective commutative algebra for polynomial rings over fields and over $\mathbb{Z}$, that is used in the specialization method of Evertse and Győry mentioned above.

We immediately deduce that equation (2.1) is effectively solvable: 
Corollary 2.1. There exists an algorithm which, for any given $f_{1}, \ldots, f_{t}$ such that $A$ is a domain, and any representatives $\tilde{a_{0}}, \ldots, \tilde{a_{n}}, \tilde{\delta}$ such that $D_{\tilde{F}}, \tilde{\delta} \notin I$, computes a finite list, consisting of one pair of representatives for each solution $(x, y)$ of (2.1).

Proof. Let $C$ be the upper bound from (2.3). Check for each pair of polynomials $\tilde{x}, \tilde{y} \in \mathbb{Z}\left[X_{1}, \ldots, X_{r}\right]$ of size at most $C$ whether $\tilde{F}(\tilde{x}, \tilde{y})-\tilde{\delta} \in I$. Then for all pairs $\tilde{x}, \tilde{y}$ passing this test, check whether they are equal modulo $I$, and keep a maximal subset of pairs that are different modulo $I$.

2.3. Hyper- and superelliptic equations. We now consider the equation

$$
F(x)=\delta y^{m} \quad \text { in } \quad x, y \in A,
$$

where

$$
F(X)=a_{0} X^{n}+a_{1} X^{n-1}+\cdots+a_{n} \in A[X]
$$

is a polynomial of degree $n$ with discriminant $D_{F} \neq 0$, and where $\delta \in A \backslash\{0\}$. We assume that either $m=2$ and $n \geq 3$, or $m \geq 3$ and $n \geq 2$. For $m=2$, equation (2.4) is called a hyperelliptic equation, while for $m \geq 3$ it is called a superelliptic equation. Choose again representatives

$$
\tilde{a_{0}}, \tilde{a_{1}}, \ldots, \tilde{a_{n}}, \tilde{\delta} \in \mathbb{Z}\left[X_{1}, \ldots, X_{r}\right]
$$

for $a_{0}, a_{1}, \ldots, a_{n}, \delta$, respectively. To guarantee that $\delta \neq 0$ and $D_{F} \neq 0$, we have to choose the representatives in such a way that $\tilde{\delta}$ and the discriminant of $\tilde{F}:=\sum_{j=0}^{n} \tilde{a_{j}} X^{n-j}$ do not belong to $I$. Let

$$
\left\{\begin{array}{l}
\max \left(\operatorname{deg} f_{1}, \ldots, \operatorname{deg} f_{t}, \operatorname{deg} \tilde{a_{0}}, \operatorname{deg} \tilde{a_{1}}, \ldots, \operatorname{deg} \tilde{a_{n}}, \operatorname{deg} \tilde{\delta}\right) \leq d \\
\max \left(h\left(f_{1}\right), \ldots, h\left(f_{t}\right), h\left(\tilde{a_{0}}\right), h\left(\tilde{a_{1}}\right), \ldots, h\left(\tilde{a_{n}}\right), h(\tilde{\delta})\right) \leq h
\end{array}\right.
$$

where $d \geq 1, h \geq 1$.

Theorem 2.2. Every solution $x, y$ of equation (2.4) has representatives $\tilde{x}, \tilde{y}$ such that

$$
s(\tilde{x}), s(\tilde{y}) \leq \exp \left(m^{3}(n d)^{\exp O(r)}(h+1)\right)
$$

Completely similarly as for Thue equations, one can determine effectively a finite list, consisting of one pair of representatives for each solution $(x, y)$ of $(2.4)$.

Our next result deals with the Schinzel-Tijdeman equation, which is (2.4) but with three unknowns $x, y \in A$ and $m \in \mathbb{Z}_{\geq 2}$. 
Theorem 2.3. Assume that in (2.4), F has non-zero discriminant and $n \geq 2$. Let $x, y \in A, m \in \mathbb{Z}_{\geq 2}$ be a solution of (2.4). Then

$$
\begin{aligned}
& m \leq \exp \left((n d)^{\exp O(r)}(h+1)\right) \\
& \quad \text { if } y \in \overline{\mathbb{Q}}, y \neq 0, y \text { is not a root of unity, } \\
& m \leq(n d)^{\exp O(r)} \text { if } y \notin \overline{\mathbb{Q}} .
\end{aligned}
$$

\section{A REDUCTION}

We shall reduce our equations to equations of the same type over an integral domain $B \supseteq A$ of a special type which is more convenient to deal with.

As before, let $A=\mathbb{Z}\left[z_{1}, \ldots, z_{r}\right]$ be an integral domain which is finitely generated over $\mathbb{Z}$ and let $K$ be the quotient field of $A$. Suppose that $K$ has transcendence degree $q \geq 0$. If $q>0$, we assume without loss of generality that $\left\{z_{1}, \ldots, z_{q}\right\}$ forms a transcendence basis of $K / \mathbb{Q}$. Write $\rho:=r-q$. We define

$$
\begin{array}{lll}
A_{0}:=\mathbb{Z}\left[z_{1}, \ldots, z_{q}\right], & K_{0}:=\mathbb{Q}\left(z_{1}, \ldots, z_{q}\right) & \text { if } q>0 \\
A_{0}:=\mathbb{Z}, & K_{0}:=\mathbb{Q} & \text { if } q=0 .
\end{array}
$$

The field $K$ is a finite extension of $K_{0}$. Further, if $q=0$, it is an algebraic number field. In case that $q>0$, for $f \in A_{0} \backslash\{0\}$ we define $\operatorname{deg} f$ and $h(f)$ to be the total degree and logarithmic height of $f$, viewed as a polynomial in the variables $z_{1}, \ldots, z_{q}$. In case that $q=0$, for $f \in A_{0} \backslash\{0\}=\mathbb{Z} \backslash\{0\}$, we put $\operatorname{deg} f:=0$ and $h(f):=\log |f|$.

We shall construct an integral extension $B$ of $A$ in $K$ such that

$$
B:=A_{0}\left[w, f^{-1}\right]
$$

where $f \in A_{0}$ and $w$ is a primitive element of $K$ over $K_{0}$ which is integral over $A_{0}$. Then we give a bound for the sizes of the solutions of our equations in $x, y \in B$.

We recall that $A \cong \mathbb{Z}\left[X_{1}, \ldots, X_{r}\right] / I$ where $I \subset \mathbb{Z}\left[X_{1}, \ldots, X_{r}\right]$ is the ideal of polynomials $f$ with $f\left(z_{1}, \ldots, z_{r}\right)=0$ and $z_{i}$ corresponds to the residue class of $X_{i}$ modulo $I$. The ideal $I$ is finitely generated. Assume that

$$
I=\left(f_{1}, \ldots, f_{t}\right),
$$


and put

$$
d_{0}:=\max \left(1, \operatorname{deg} f_{1}, \ldots, \operatorname{deg} f_{t}\right), \quad h_{0}:=\max \left(1, h\left(f_{1}\right), \ldots, h\left(f_{t}\right)\right) .
$$

Proposition 3.1. (i) There is a $w \in A$ such that $K=K_{0}(w)$, $w$ is integral over $A_{0}$ and $w$ has minimal polynomial

$$
\mathcal{F}(X)=X^{D}+\mathcal{F}_{1} X^{D-1}+\cdots+\mathcal{F}_{D} \in A_{0}[X]
$$

over $K_{0}$ such that $D \leq d_{0}^{\rho}$ and

$$
\operatorname{deg} \mathcal{F}_{k} \leq\left(2 d_{0}\right)^{\exp O(r)}, \quad h\left(\mathcal{F}_{k}\right) \leq\left(2 d_{0}\right)^{\exp O(r)}\left(h_{0}+1\right)
$$

for $k=1, \ldots, D$.

(ii) Let $\alpha_{1}, \ldots, \alpha_{k} \in K^{*}$ and suppose that the pairs $u_{i}, v_{i} \in \mathbb{Z}\left[X_{1}, \ldots, X_{r}\right]$, $v_{i} \notin I$ represent $\alpha_{i}$ for $i=1, \ldots, k$, respectively. Put

$$
\begin{aligned}
& d^{* *}:=\max \left(d_{0}, \operatorname{deg} u_{1}, \operatorname{deg} v_{1}, \ldots, \operatorname{deg} u_{k}, \operatorname{deg} v_{k}\right), \\
& h^{* *}:=\max \left(h_{0}, h\left(u_{1}\right), h\left(v_{1}\right), \ldots, h\left(u_{k}\right), h\left(v_{k}\right)\right) .
\end{aligned}
$$

Then there is a non-zero $f \in A_{0}$ such that

$$
\begin{aligned}
& A \subseteq A_{0}\left[w, f^{-1}\right], \\
& \alpha_{1}, \ldots, \alpha_{k} \in A_{0}\left[w, f^{-1}\right]^{*}
\end{aligned}
$$

and

(3.5) $\operatorname{deg} f \leq(k+1)\left(2 d^{* *}\right)^{\exp O(r)}, \quad h(f) \leq(k+1)\left(2 d^{* *}\right)^{\exp O(r)}\left(h^{* *}+1\right)$.

Proof. For (i) see Evertse and Győry [9], Proposition 3.4 and Lemma 3.2, (i), and for (ii) see [9], Lemma 3.6.

We shall use Proposition 3.1, (ii) in a special case. To state it, we introduce some further notation and prove a lemma.

We recall that $a_{0}, a_{1}, \ldots, a_{n} \in A$ are the coefficients of the binary form $F(X, Y)$, resp. of the polynomial $F(X)$ in Sections 2.2 resp. 2.3, and $\tilde{a}_{0}, \tilde{a}_{1}, \ldots, \tilde{a}_{n}$ denote their representatives satisfying (2.2) resp. (2.5). This implies that $d_{0} \leq d, h_{0} \leq h$, and that $\tilde{a}_{i}$ has total degree $\leq d$ and logarithmic height $\leq h$ for $i=0, \ldots, n$. Denote by $\tilde{F}$ the binary form $F(X, Y)$ resp. the polynomial $F(X)$ with coefficients $a_{0}, a_{1}, \ldots, a_{n}$ replaced by $\tilde{a}_{0}, \tilde{a}_{1}, \ldots, \tilde{a}_{n}$, and by $D_{\tilde{F}}$ the discriminant of $\tilde{F}$. In view of the assumption $D_{F} \neq 0$ we have $D_{\tilde{F}} \notin I$. 
Keeping the notation and assumptions of Sections 2.2 resp. 2.3, we have the following lemma.

Lemma 3.2. For the discriminant $D_{\tilde{F}}$ the following statements are true:

$$
\begin{aligned}
& \operatorname{deg} D_{\tilde{F}} \leq(2 n-2) d, \\
& h\left(D_{\tilde{F}}\right) \leq(2 n-2)\left(\log \left(2 n^{2}\left(\begin{array}{c}
d+r \\
r
\end{array}\right)\right)+h\right) .
\end{aligned}
$$

Proof. Recall that the discriminant $D_{\tilde{F}}$ can be expressed as

$$
D(\tilde{F})= \pm\left|\begin{array}{ccccccc}
\tilde{a}_{0} & \tilde{a}_{1} & \cdots & \cdots & \tilde{a}_{n} & & \\
& \ddots & & & & \ddots & \\
& & \tilde{a}_{0} & \tilde{a}_{1} & \cdots & \cdots & \tilde{a}_{m} \\
\tilde{a}_{1} & 2 \tilde{a}_{2} & \cdots & n \tilde{a}_{n} & & & \\
n \tilde{a}_{0} & (n-1) \tilde{a}_{1} & \cdots & \tilde{a}_{n-1} & & & \\
& \ddots & & & \ddots & & \\
& & \ddots & & & \ddots & \\
& & & n \tilde{a}_{0} & (n-1) \tilde{a}_{1} & \cdots & \tilde{a}_{n-1}
\end{array}\right|,
$$

with on the first $n-2$ rows of the determinant $\tilde{a_{0}}, \ldots, \tilde{a_{n}}$, on the $(n-1)$-st row $\tilde{a}_{1}, 2 \tilde{a}_{2}, \ldots, n \tilde{a}_{n}$, and on the last $n-1$ rows $n \tilde{a}_{0}, \ldots, \tilde{a}_{n-1}$. This implies at once (3.6).

To prove (3.7), we use the length $L(P)$ of a polynomial $P \in \mathbb{Z}\left[X_{1}, \ldots, X_{r}\right]$, that is the sum of the absolute values of the coefficients of $P$. It is known and easily seen that if $P, Q \in \mathbb{Z}\left[X_{1}, \ldots, X_{r}\right]$ then $L(P+Q)$ and $L(P Q)$ do not exceed $L(P)+L(Q)$ and $L(P) L(Q)$, respectively (see e.g. Waldschmidt [23], p.76).

We have

$$
L\left(\tilde{a}_{i}\right) \leq\left(\begin{array}{c}
d+r \\
r
\end{array}\right) H \quad \text { with } \quad H=\exp h \quad \text { for } i=0, \ldots, n .
$$

By applying these facts to (3.8), we obtain

$$
L\left(D_{\tilde{F}}\right) \leq(2 n-2) !\left(n\left(\begin{array}{c}
d+r \\
r
\end{array}\right) H\right)^{2 n-2} .
$$

Together with $h\left(D_{\tilde{F}}\right) \leq \log L\left(D_{\tilde{F}}\right)$ this implies (3.7). 
We now apply Proposition 3.1, (ii) to the numbers $\alpha_{1}=\delta, \alpha_{2}=\delta^{-1}, \alpha_{3}=$ $D_{F}$ and $\alpha_{4}=D_{F}^{-1}$. Then the pairs $(\tilde{\delta}, 1),(1, \tilde{\delta}),\left(D_{\tilde{F}}, 1\right),\left(1, D_{\tilde{F}}\right)$ represent the numbers $\alpha_{i}, i=1, \ldots, 4$. Using the upper bounds for $\operatorname{deg} D_{\tilde{F}}, h\left(D_{\tilde{F}}\right)$ implied by Lemma 3.2 as well as the upper bounds $\operatorname{deg} \tilde{\delta} \leq d, h(\tilde{\delta}) \leq h$ implied by (2.2), (2.5), we get immediately from Proposition 3.1, (ii) the following.

Proposition 3.3. There is a non-zero $f \in A_{0}$ such that

$$
A \subseteq A_{0}\left[w, f^{-1}\right], \quad \delta, D_{F} \in A_{0}\left[w, f^{-1}\right]^{*}
$$

and

$$
\operatorname{deg} f \leq(n d)^{\exp O(r)}, \quad h(f) \leq(n d)^{\exp O(r)}(h+1) .
$$

In the case $q>0, z_{1}, \ldots, z_{q}$ are algebraically independent. Thus, for $q \geq 0, A_{0}$ is a unique factorization domain, and hence the greatest common divisor of a finite set of elements of $A_{0}$ is well defined and up to sign uniquely determined. We associate with every element $\alpha \in K$ the up to sign unique tuple $P_{\alpha, 0}, \ldots, P_{\alpha, D-1}, Q_{\alpha}$ of elements of $A_{0}$ such that

$$
\alpha=Q_{\alpha}^{-1} \sum_{j=0}^{D-1} P_{\alpha, j} w^{j} \quad \text { with } \quad Q_{\alpha} \neq 0, \quad \operatorname{gcd}\left(P_{\alpha, 0}, \ldots, P_{\alpha, D-1}, Q_{\alpha}\right)=1 .
$$

We put

$$
\left\{\begin{array}{l}
\overline{\operatorname{deg}} \alpha:=\max \left(\operatorname{deg} P_{\alpha, 0}, \ldots, \operatorname{deg} P_{\alpha, D-1}, \operatorname{deg} Q_{\alpha}\right) \\
\bar{h}(\alpha):=\max \left(h\left(P_{\alpha, 0}\right), \ldots, h\left(P_{\alpha, D-1}\right), h\left(Q_{\alpha}\right)\right)
\end{array}\right.
$$

where as usual, $\operatorname{deg} P, h(P)$ denote the total degree and logarithmic height of a polynomial $P$ with rational integral coefficients. Thus for $q=0$ we have $\overline{\operatorname{deg}} \alpha=0$ and $\bar{h}(\alpha)=\log \max \left(\left|P_{\alpha, 0}\right|, \ldots,\left|P_{\alpha, D-1}\right|,\left|Q_{\alpha}\right|\right)$.

Lemma 3.4. Let $\alpha \in K^{*}$ and let $(a, b)$ be a pair of representatives for $\alpha$ with $a, b \in \mathbb{Z}\left[X_{1}, \ldots, X_{r}\right], b \notin I$. Put

$$
d^{*}=\max \left(d_{0}, \operatorname{deg} a, \operatorname{deg} b\right) \quad \text { and } \quad h^{*}:=\max \left(h_{0}, h(a), h(b)\right) .
$$

Then

$$
\overline{\operatorname{deg}} \alpha \leq\left(2 d^{*}\right)^{\exp O(r)}, \quad \bar{h}(\alpha) \leq\left(2 d^{*}\right)^{\exp O(r)}\left(h^{*}+1\right) .
$$

Proof. This is Lemma 3.5 in Evertse and Győry [9]. 
Lemma 3.5. Let $\alpha$ be a nonzero element of $A$, and put

$$
\widehat{d}:=\max \left(d_{0}, \overline{\operatorname{deg}} \alpha\right), \quad \widehat{h}:=\max \left(h_{0}, \bar{h}(\alpha)\right) .
$$

Then $\alpha$ has a representative $\tilde{\alpha} \in \mathbb{Z}\left[X_{1}, \ldots, X_{r}\right]$ such that

$$
\left\{\begin{array}{l}
\operatorname{deg} \tilde{\alpha} \leq(2 \widehat{d})^{\exp O\left(r \log ^{*} r\right)}(\widehat{h}+1) \\
h(\tilde{\alpha}) \leq(2 \widehat{d})^{\exp O\left(r \log ^{*} r\right)}(\widehat{h}+1)^{r+1}
\end{array}\right.
$$

Proof. This is a special case of Lemma 3.7 of Evertse and Györy [9] with the choice $\lambda=1$ and $a=b=1$. The proof of this lemma is based on work of Aschenbrenner [1].

3.1. Thue equations. Recall that $A_{0}=\mathbb{Z}\left[z_{1}, \ldots, z_{q}\right], K_{0}=\mathbb{Q}\left(z_{1}, \ldots, z_{q}\right)$ if $q>0$, and $A_{0}=\mathbb{Z}, K_{0}=\mathbb{Q}$ if $q=0$, and that in the case $q=0$ total degrees and $\overline{\mathrm{deg}}$-s are always zero. Further, we have

$$
F(X, Y)=a_{0} X^{n}+a_{1} X^{n-1} Y+\cdots+a_{n} Y^{n} \in A[X, Y]
$$

with $n \geq 3$ and with discriminant $D_{F} \neq 0$, and $\delta \in A \backslash\{0\}$. Recall that for $a_{0}, a_{1}, \ldots, a_{n}, \delta$ we have chosen representatives $\tilde{a_{0}}, \tilde{a_{1}}, \ldots, \tilde{a_{n}}, \tilde{\delta} \in$ $\mathbb{Z}\left[X_{1}, \ldots, X_{r}\right]$ satisfying $(2.2)$.

Theorem 2.1 will be deduced from the following Proposition, which makes sense also if $q=0$. The proof of this proposition is given in Sections 4-6.

Proposition 3.6. Let $w$ and $f$ be as in Propositions 3.1, (i) and 3.3, respectively, with the properties specified there, and consider the integral domain

$$
B:=A_{0}\left[f^{-1}, w\right]
$$

Then for the solutions $x, y$ of the equation

$$
F(x, y)=\delta \quad \text { in } \quad x, y \in B
$$

we have

$$
\begin{aligned}
& \overline{\operatorname{deg}} x, \overline{\operatorname{deg}} y \leq(n d)^{\exp O(r)}, \\
& \bar{h}(x), \bar{h}(y) \leq \exp \left(n !(n d)^{\exp O(r)}(h+1)\right) .
\end{aligned}
$$

We now deduce Theorem 2.1 from Proposition 3.6. 
Proof of Theorem 2.1. Let $x, y$ be a solution of equation (2.1). In view of (3.9) $x, y$ is also a solution in $B=A_{0}\left[f^{-1}, w\right]$, where $f, w$ satisfy the conditions specified in Propositions 3.1, (i) and 3.3, respectively. Then by Proposition 3.6, the inequalities (3.16) and (3.17) hold. Applying now Lemma 3.5 to $x$ and $y$, we infer that $x, y$ have representatives $\tilde{x}, \tilde{y}$ in $Z\left[X_{1}, \ldots, X_{r}\right]$ with (2.3).

3.2. Hyper- and superelliptic equations. Recall that the polynomial

$$
F(X)=a_{0} X^{n}+a_{1} X^{n-1}+\cdots+a_{n} \in A[X]
$$

has discriminant $D_{F} \neq 0$, that $\delta \in A \backslash\{0\}$, and that for $a_{0}, a_{1}, \ldots, a_{n}, \delta$ we have chosen representatives $\tilde{a_{0}}, \tilde{a_{1}}, \ldots, \tilde{a_{n}}, \tilde{\delta} \in \mathbb{Z}\left[X_{1}, \ldots, X_{r}\right]$ satisfying $(2.5)$.

Theorem 2.2 will be deduced from the following Proposition, which has a meaning also if $q=0$. Similarly as its analogue for Thue equations, its proof is given in Sections 4-6.

Proposition 3.7. Let $w$ and $f$ be as in Propositions 3.1, (i) and 3.3, respectively, with the properties specified there, and consider the domain

$$
B:=A_{0}\left[f^{-1}, w\right] .
$$

Further, let $m$ be an integer $\geq 2$, and assume that $n \geq 3$ if $m=2$ and $n \geq 2$ if $m \geq 3$. Then for the solutions $x, y$ of the equation

$$
F(x)=\delta y^{m} \quad \text { in } \quad x, y \in B
$$

we have

$$
\begin{aligned}
& \overline{\operatorname{deg}} x, m \overline{\operatorname{deg}} y \leq(n d)^{\exp O(r)} \\
& \bar{h}(x), \bar{h}(y) \leq \exp \left(m^{3}(n d)^{\exp O(r)}(h+1)\right)
\end{aligned}
$$

We now deduce Theorem 2.2 from Proposition 3.7.

Proof of Theorem 2.2. Let $x, y$ be a solution of equation (2.4). In view of (3.9) $x, y$ is also a solution in $B=A_{0}\left[f^{-1}, w\right]$, where $f, w$ satisfy the conditions specified in Propositions 3.1, (i) and 3.3, respectively. Then by Proposition 3.7, (3.19) and (3.20) hold. Applying now Lemma 3.5 to $x$ and $y$, we infer that $x, y$ have representatives $\tilde{x}, \tilde{y}$ in $Z\left[X_{1}, \ldots, X_{r}\right]$ with $(2.6)$. 
Proposition 3.8. Suppose that equation (3.18) has a solution $x \in B, y \in$ $B \cap \overline{\mathbb{Q}}$ and that also $y \neq 0$ and $y$ is not a root of unity. Then

$$
m \leq \exp \left((n d)^{\exp O(r)}(h+1)\right) .
$$

Proof of Theorem 2.3. Let $x, y \in A, m \in \mathbb{Z}_{\geq 2}$ be a solution of equation (2.4). First let $y \notin \overline{\mathbb{Q}}$. Then $\overline{\operatorname{deg}} y \geq 1$, and together with (3.19) this implies (2.8). Next, let $y \in \overline{\mathbb{Q}}$. Then Proposition 3.8 gives at once (2.7).

The proof of Proposition 3.8 is a combination of results from Sections 4-6. It is completed at the end of Section 6 .

\section{Bounding THE DEGREE}

In this section we shall prove (3.16) of Proposition 3.6 and (3.19) of Proposition 3.7.

We recall some results on function fields in one variable. Let $\mathbb{k}$ be an algebraically closed field of characteristic $0, z$ a transcendental element over $\mathbb{k}$ and $M$ a finite extension of $\mathbb{k}(z)$. Denote by $g_{M / \mathbb{k}}$ the genus of $M$, and by $\mathcal{M}_{M}$ the collection of valuations of $M / \mathbb{k}$, these are the discrete valuations of $M$ with value group $\mathbb{Z}$ which are trivial on $\mathbb{k}$. Recall that these valuations satisfy the sum formula

$$
\sum_{v \in \mathcal{M}_{M}} v(\alpha)=0 \quad \text { for } \quad \alpha \in M^{*} .
$$

For a finite subset $S$ of $\mathcal{M}_{M}$, an element $\alpha \in M$ is called an $S$-integer if $v(\alpha) \geq 0$ for all $v \in \mathcal{M}_{M} \backslash S$. The $S$-integers form a ring in $M$, denoted by $\mathcal{O}_{S}$. The (homogeneous) height of $\mathbf{a}=\left(\alpha_{1}, \ldots, \alpha_{l}\right) \in M^{l}$ relative to $M / \mathbb{k}$ is defined by

$$
H_{M}(\mathbf{a})=H_{M}\left(\alpha_{1}, \ldots, \alpha_{l}\right):=-\sum_{v \in \mathcal{M}_{M}} \min \left(v\left(\alpha_{1}\right), \ldots, v\left(\alpha_{l}\right)\right),
$$

and we define the height $H_{M}(f)$ of a polynomial $f \in M[X]$ by the height of the vector defined by the coefficients of $f$. Further, we shall write $H_{M}(1, \mathbf{a}):=H_{M}\left(1, \alpha_{1}, \ldots, \alpha_{l}\right)$. We note that

$$
H_{M}\left(\alpha_{i}\right) \leq H_{M}(\mathbf{a}) \leq H_{M}\left(\alpha_{1}\right)+\cdots+H_{M}\left(\alpha_{l}\right), \quad i=1, \ldots, l .
$$

By the sum formula,

$$
H_{M}(\alpha \mathbf{a})=H_{M}(\mathbf{a}) \quad \text { for } \quad \alpha \in M^{*} .
$$


The height of $\alpha \in M$ relative to $M / \mathbb{k}$ is defined by

$$
H_{M}(\alpha):=H_{M}(1, \alpha)=-\sum_{v \in \mathcal{M}_{M}} \min (0, v(\alpha)) .
$$

It is clear that $H_{M}(\alpha)=0$ if and only if $\alpha \in \mathbb{k}$. Using the sum formula, it is easy to prove that the height has the properties

$$
\begin{aligned}
& H_{M}\left(\alpha^{l}\right)=|l| H_{M}(\alpha), \\
& H_{M}(\alpha+\beta) \leq H_{M}(\alpha)+H_{M}(\beta), \quad H_{M}(\alpha \beta) \leq H_{M}(\alpha)+H_{M}(\beta)
\end{aligned}
$$

for all non-zero $\alpha, \beta \in M$ and for every integer $l$.

If $L$ is a finite extension of $M$, we have

$$
H_{L}\left(\alpha_{0}, \ldots, \alpha_{l}\right)=[L: M] H_{M}\left(\alpha_{0}, \ldots, \alpha_{l}\right) \quad \text { for } \quad \alpha_{0}, \ldots, \alpha_{l} \in M .
$$

By $\operatorname{deg} f$ we denote the total degree of $f \in \mathbb{k}[z]$. Then for $f_{0}, \ldots, f_{l} \in \mathbb{k}[z]$ with $\operatorname{gcd}\left(f_{0}, \ldots, f_{l}\right)=1$ we have

$$
H_{\mathbb{R}[z]}\left(f_{0}, \ldots, f_{l}\right)=\max \left(\operatorname{deg} f_{0}, \ldots, \operatorname{deg} f_{l}\right) .
$$

Lemma 4.1. Let $\alpha_{1}, \ldots, \alpha_{l} \in M$ and suppose that

$$
X^{l}+f_{1} X^{l-1}+\cdots+f_{l}=\left(X-\alpha_{1}\right) \ldots\left(X-\alpha_{l}\right)
$$

for certain $f_{1}, \ldots f_{l} \in \mathbb{k}[z]$. Then

$$
[M: \mathbb{k}(z)] \max \left(\operatorname{deg} f_{1}, \ldots, \operatorname{deg} f_{l}\right)=\sum_{i=1}^{l} H_{M}\left(\alpha_{i}\right) .
$$

Proof. This is Lemma 4.1 in Evertse and Győry [9].

Lemma 4.2. Let

$$
F=f_{0} X^{l}+f_{1} X^{l-1}+\cdots+f_{l} \in M[X]
$$

be a polynomial with $f_{0} \neq 0$ and with non-zero discriminant. Let $L$ be the splitting field over $M$ of $F$. Then

$$
g_{L / \mathbb{k}} \leq[L: M] \cdot\left(g_{M / \mathbb{k}}+l H_{M}(F)\right) .
$$

In particular, if $M=\mathbb{k}(z)$ and $f_{0}, \ldots, f_{l} \in \mathbb{k}[z]$, we have

$$
g_{L / \mathbb{k}} \leq[L: M] \cdot l \max \left(\operatorname{deg} f_{0}, \ldots, \operatorname{deg} f_{l}\right) .
$$


Proof. The second assertion follows by combining the first assertion with (4.5). We now prove the first assertion. Our proof is a generalization of that of Lemma H of Schmidt [18].

For $v \in \mathcal{M}_{M}$, put $v(F):=\min \left(v\left(f_{0}\right), \ldots, v\left(f_{l}\right)\right)$. Let $D_{F}$ denote the discriminant of $F$. Since $D_{F}$ is a homogeneous polynomial of degree $2 l-2$ in $f_{0}, \ldots, f_{l}$, we have

$$
v\left(D_{F}\right) \geq(2 l-2) v(F) .
$$

Let $S$ be the set of $v \in \mathcal{M}_{M}$ with $v\left(f_{0}\right)>v(F)$ or $v\left(D_{F}\right)>(2 l-2) v(F)$. We show that $L / M$ is unramified over every valuation $v \in \mathcal{M}_{M} \backslash S$.

Take $v \in \mathcal{M}_{M} \backslash S$. Let

$$
O_{v}:=\{x \in M: v(x) \geq 0\}, \quad m_{v}:=\{x \in M: v(x)>0\}
$$

denote the local ring at $v$, and the maximal ideal of $O_{v}$, respectively. The residue class field $O_{v} / m_{v}$ is equal to $\mathbb{k}$ since $\mathbb{k}$ is algebraically closed. Let $\varphi_{v}: O_{v} \rightarrow \mathbb{k}$ denote the canonical homomorphism.

Without loss of generality, we assume $v(F)=0$. Then $v\left(f_{0}\right)=0$, $v\left(D_{F}\right)=0$. Let $\varphi_{v}(F):=\sum_{j=0}^{l} \varphi_{v}\left(f_{j}\right) X^{l-j}$. Then $\varphi_{v}\left(f_{0}\right) \neq 0$ and $\varphi_{v}(F)$ has discriminant $\varphi_{v}\left(D_{F}\right) \neq 0$. Since $D_{F} \neq 0$, the polynomial $F$ has $l$ distinct zeros in $L, \alpha_{1}, \ldots, \alpha_{l}$, say. Further, $\varphi_{v}(F)$ has $l$ distinct zeros in $\mathbb{k}$, $a_{1}, \ldots, a_{l}$, say.

Denote by $\Sigma_{l}$ the permutation group on $(1, \ldots, l)$. Choose $c_{1}, \ldots, c_{l} \in \mathbb{k}$, such that the numbers

$$
\alpha_{\sigma}:=c_{1} \alpha_{\sigma(1)}+\cdots+c_{l} \alpha_{\sigma(l)} \quad\left(\sigma \in \Sigma_{l}\right)
$$

are all distinct, and the numbers

$$
a_{\sigma}:=c_{1} a_{\sigma(1)}+\cdots+c_{l} a_{\sigma(l)} \quad\left(\sigma \in \Sigma_{l}\right)
$$

are all distinct. Let $\alpha:=c_{1} \alpha_{1}+\cdots+c_{l} \alpha_{l}$. Then $L=M(\alpha)$, and the monic minimal polynomial of $\alpha$ over $M$ divides $G:=\prod_{\sigma \in \Sigma_{l}}\left(X-\alpha_{\sigma}\right)$ which by the theorem of symmetric functions belongs to $M[X]$. The image of $G$ under $\varphi_{v}$ is $\prod_{\sigma \in \Sigma_{l}}\left(X-a_{\sigma}\right)$ and this has only simple zeros. This implies that $L / M$ is unramified at $v$.

For $v \in \mathcal{M}_{M}$ and any valuation $\in \mathcal{M}_{L}$ above $v$, denote by $e(V \mid v)$ the ramification index of $V$ over $v$. Recall that $\sum_{V \mid v} e(V \mid v)=[L: M]$, where 
the sum is taken over all valuations of $L$ lying above $v$. Now the RiemannHurwitz formula implies that

$$
\begin{aligned}
2 g_{L / \mathbb{k}}-2 & =[L: M]\left(2 g_{K}-2\right)+\sum_{v \in S} \sum_{V \mid v}(e(V \mid v)-1) \\
& \leq[L: M]\left(2 g_{K}-2+|S|\right),
\end{aligned}
$$

where $|S|$ denotes the cardinality of $S$. It remains to estimate $|S|$. By the sum formula and (4.6) we have

$$
\begin{aligned}
|S| & \leq \sum_{v \in S}\left(\left(v\left(f_{0}\right)-v(F)\right)+\left(v\left(D_{F}\right)-(2 l-2) v(F)\right)\right) \\
& =-\sum_{v \in S}(2 l-1) v(F)-\sum_{v \in \mathcal{M}_{M} \backslash S} v\left(f_{0}\right)-\sum_{v \in \mathcal{M}_{M} \backslash S} v\left(D_{F}\right) \\
& \leq-(2 l-1) \sum_{v \in \mathcal{M}_{M}} v(F)=(2 l-1) H_{M}(F) .
\end{aligned}
$$

By inserting this into (4.7) we arrive at an inequality which is stronger than what we wanted to prove.

In the sequel we keep the notation of Proposition 3.1. To prove (3.16) and (3.19) we may suppose that $q>0$ since the case $q=0$ is trivial. Let again $K_{0}:=\mathbb{Q}\left(z_{1}, \ldots, z_{q}\right), K:=K_{0}(w), A_{0}:=\mathbb{Z}\left[z_{1}, \ldots, z_{q}\right], B:=$ $\mathbb{Z}\left[z_{1}, \ldots, z_{q}, f^{-1}, w\right]$ with $f, w$ specified in Propositions 3.1 (i) and 3.3.

Fix $i \in\{1, \ldots, q\}$. Let $\mathbb{k}_{i}:=\mathbb{Q}\left(z_{1}, \ldots, z_{i-1}, z_{i+1}, \ldots, z_{q}\right)$ and $\overline{\mathbb{k}}_{i}$ its algebraic closure. Then $A_{0}$ is contained in $\overline{\mathbb{k}}_{i}\left[z_{i}\right]$. Denote by $w^{(1)}:=w, \ldots, w^{(D)}$ the conjugates of $w$ over $K_{0}$. Let $M_{i}$ denote the splitting field of the polynomial $X^{D}+\mathcal{F}_{1} X^{D-1}+\cdots+\mathcal{F}_{D}$ over $\overline{\mathbb{k}}_{i}\left(z_{i}\right)$, that is

$$
M_{i}:=\overline{\mathbb{k}}_{i}\left(z_{i}, w^{(1)}, \ldots, w^{(D)}\right) .
$$

Then

$$
B_{i}:=\overline{\mathbb{k}}_{i}\left[z_{i}, f^{-1}, w^{(1)}, \ldots, w^{(D)}\right]
$$

is a subring of $M_{i}$ which contains $B=\mathbb{Z}\left[z_{1}, \ldots, z_{q}, f^{-1}, w\right]$ as a subring. Let $\Delta_{i}:=\left[M_{i}: \overline{\mathbb{k}}_{i}\left(z_{i}\right)\right]$. Further, let $g_{M_{i}}$ denote the genus of $M_{i} / \overline{\mathbb{k}}_{i}$, and $H_{M_{i}}$ the height taken with respect to $M_{i} / \overline{\mathbb{k}}_{i}$. Put

$$
d_{1}:=\max \left(d_{0}, \operatorname{deg} f, \operatorname{deg} \mathcal{F}_{1}, \ldots, \operatorname{deg} \mathcal{F}_{D}\right) .
$$

We mention that in view of Propositions 3.1, 3.3,

$$
d_{1} \leq(n d)^{\exp O(r)} .
$$


Lemma 4.3. Let $\alpha \in K^{*}$ and denote by $\alpha^{(1)}, \ldots, \alpha^{(D)}$ the conjugates of $\alpha$ corresponding to $w^{(1)}, \ldots, w^{(D)}$. Then

$$
\overline{\operatorname{deg}} \alpha \leq q D d_{1}+\sum_{i=1}^{q} \Delta_{i}^{-1} \sum_{j=1}^{D} H_{M_{i}}\left(\alpha^{(j)}\right) .
$$

Proof. This is Lemma 4.4 in Evertse and Győry [9].

Conversely, we have the following:

Lemma 4.4. Let $\alpha \in K^{*}$ and $\alpha^{(1)}, \ldots, \alpha^{(D)}$ be as in Lemma 4.3. Then we have

$$
\max _{i, j} H_{M_{i}}\left(\alpha^{(j)}\right) \leq \Delta_{i}\left(2 D \overline{\operatorname{deg}} \alpha+\left(2 d_{0}\right)^{\exp O(r)}\right)
$$

Proof. Consider the representation of the form (3.11) of $\alpha$. Since $P_{\alpha, k}, Q \in$ $K_{0}$, we have

$$
\alpha^{(j)}=\sum_{k=0}^{D-1} \frac{P_{\alpha, k}}{Q}\left(w^{(j)}\right)^{k} \quad \text { for } \quad j=1, \ldots, D .
$$

In view of (4.3) it follows that

$$
H_{M_{i}}\left(\alpha^{(j)}\right) \leq \sum_{k=0}^{D-1} H_{M_{i}}\left(\frac{P_{\alpha, k}}{Q}\right)+\sum_{k=0}^{D-1} k H_{M_{i}}\left(w^{(j)}\right) .
$$

But we have

$$
\begin{aligned}
H_{M_{i}}\left(\frac{P_{\alpha, k}}{Q}\right) & \leq \Delta_{i} H_{\mathbb{k}_{i}(z)}\left(\frac{P_{\alpha, k}}{Q}\right) \leq \Delta_{i}\left(\operatorname{deg}_{z_{i}} P_{\alpha, k}+\operatorname{deg}_{z_{i}} Q\right) \\
& \leq \Delta_{i}\left(\operatorname{deg} P_{\alpha, k}+\operatorname{deg} Q\right) \leq 2 \Delta_{i} \overline{\operatorname{deg}} \alpha
\end{aligned}
$$

Further, applying Lemma 4.1 with $M_{i}, w^{(1)}, \ldots, w^{(D)}$ instead of $M, \alpha_{1}, \ldots, \alpha_{l}$, we get

$$
\begin{aligned}
H_{M_{i}}\left(w^{(j)}\right) & \leq \Delta_{i} \max _{1 \leq j \leq D}\left(\operatorname{deg}_{z_{i}} \mathcal{F}_{j}\right) \\
& \leq \Delta_{i} \max _{1 \leq j \leq D}\left(\operatorname{deg} \mathcal{F}_{j}\right) \leq \Delta_{i}\left(2 d_{0}\right)^{\exp O(r)} .
\end{aligned}
$$

Now using the fact that $D \leq d_{0}^{\rho} \leq d_{0}^{r-1}$, (4.11), (4.12) and (4.13) imply (4.10). 
4.1. Thue equations. As before, $\mathbb{k}$ is an algebraically closed field of characteristic $0, z$ a transcendental element over $\mathbb{k}$ and $M$ a finite extension of $\mathbb{k}(z)$. Further, $g_{M / \mathbb{k}}$ denotes the genus of $M, \mathcal{M}_{M}$ the collection of valuations of $M / \mathbb{k}$, and for a finite subset $S$ of $\mathcal{M}_{M}, \mathcal{O}_{S}$ denotes the ring of $S$-integers in $M$. We denote by $|S|$ the cardinality of $S$.

Consider now the Thue equation

$$
F(x, y)=1 \quad \text { in } \quad x, y \in \mathcal{O}_{S},
$$

where $F$ is a binary form of degree $n \geq 3$ with coefficients in $M$ and with non-zero discriminant.

Proposition 4.5. Every solution $x, y \in \mathcal{O}_{S}$ of (4.14) satisfies

$$
\max \left(H_{M}(x), H_{M}(y)\right) \leq 89 H_{M}(F)+212 g_{M / \mathbb{k}}+|S|-1 .
$$

Proof. This is Theorem 1, (ii) of Schmidt [18].

We note that from Mason's fundamental inequality concerning $S$-unit equations over function fields (see Mason [16]) one could deduce (4.15) with smaller constants than 89 and 212. However, this is irrelevant for the bounds in $(2.3)$.

Now we use Proposition 4.5 to prove the statement (3.16) of Proposition 3.6 .

Proof of (3.16). We denote by $w^{(1)}:=w, \ldots, w^{(D)}$ the conjugates of $w$ over $K_{0}$, and for $\alpha \in K$ we denote by $\alpha^{(1)}, \ldots, \alpha^{(D)}$ the conjugates of $\alpha$ corresponding to $w^{(1)}, \ldots, w^{(D)}$.

Next, for $i=1, \ldots, n$ we put $\mathbb{k}_{i}:=\mathbb{Q}\left(z_{1}, \ldots, z_{i-1}, z_{i+1}, \ldots, z_{q}\right)$ and denote by $\overline{\mathbb{k}}_{i}$ its algebraic closure. Further, $M_{i}$ denotes the splitting field of the polynomial $X^{D}+\mathcal{F}_{1} X^{D-1}+\cdots+\mathcal{F}_{D}$ over $\overline{\mathbb{k}}_{i}\left(z_{i}\right)$, we put $\Delta_{i}:=\left[M_{i}: \overline{\mathbb{k}}_{i}\left(z_{i}\right)\right]$ and define

$$
S_{i}:=\left\{v \in \mathcal{M}_{M_{i}}: v\left(z_{i}\right)<0 \text { or } v(f)>0\right\} .
$$

The conjugates $w^{(j)}(j=1, \ldots, D)$ lie in $M_{i}$ and are all integral over $\mathbb{k}_{i}\left[z_{i}\right]$. Hence they belong to $\mathcal{O}_{S_{i}}$. Further, $f^{-1} \in \mathcal{O}_{S_{i}}$. Consequently, if $\alpha \in B=$ $A_{0}\left[f^{-1}, w\right]$, then $\alpha^{(j)} \in \mathcal{O}_{S_{i}}$ for $j=1, \ldots, D, i=1, \ldots, q$.

Let $x, y$ be a solution of equation (3.15). Put $F^{\prime}:=\delta^{-1} F$, and let $F^{\prime(j)}$ be the binary form obtained by taking the $j$-th conjugates of the coefficients of 
$F^{\prime}$. Let $j \in\{1, \ldots, D\}, i \in\{1, \ldots, q\}$. Then clearly, $F^{\prime(j)} \in M_{i}[X, Y]$, and

$$
F^{(j)}\left(x^{(j)}, y^{(j)}\right)=1, \quad x^{(j)}, y^{(j)} \in \mathcal{O}_{S_{i}} .
$$

So by Proposition 4.5 we obtain that

$$
\max \left(H_{M_{i}}\left(x^{(j)}\right), H_{M_{i}}\left(y^{(j)}\right)\right) \leq 89 H_{M_{i}}\left(F^{(j)}\right)+212 g_{M_{i}}+\left|S_{i}\right|-1 .
$$

We estimate the various parameters in this bound. We start with $H_{M_{i}}\left(F^{\prime(j)}\right)$. We recall that $F^{\prime}(X, Y)=\delta^{-1}\left(a_{0} X^{n}+a_{1} X^{n-1} Y+\cdots+a_{n} Y^{n}\right)$. Using (4.2), (4.1) and Lemma 4.4 we infer that

$$
\begin{aligned}
H_{M_{i}}\left(F^{(j)}\right) & =H_{M_{i}}\left(a_{0}^{(j)}, \ldots, a_{n}^{(j)}\right) \leq H_{M_{i}}\left(a_{0}^{(j)}\right)+\cdots+H_{M_{i}}\left(a_{n}^{(j)}\right) \\
& \leq \Delta_{i}\left(2 D\left(\overline{\operatorname{deg}} a_{0}+\cdots+\overline{\operatorname{deg}} a_{n}\right)+n\left(2 d_{0}\right)^{\exp O(r)}\right) .
\end{aligned}
$$

By Lemma 3.4 we have

$$
\overline{\operatorname{deg}} a_{i} \leq\left(2 d^{*}\right)^{\exp O(r)} \quad \text { for } \quad i=0, \ldots, n,
$$

where $d^{*}:=\max \left(d_{0}, \operatorname{deg} \tilde{a}_{i}\right) \leq d$. Further, we have $d_{0} \leq d, D \leq d_{0}^{r-q} \leq d^{r}$. Thus we obtain that

$$
\begin{aligned}
H_{M_{i}}\left(F^{\prime(j)}\right) & \leq \Delta_{i}\left(2 D(n+1)(2 d)^{\exp O(r)}+n(2 d)^{\exp O(r)}\right) \\
& \leq \Delta_{i}(n d)^{\exp O(r)} .
\end{aligned}
$$

Next, we estimate the genus. Using Lemma 4.2 with $F(X)=\mathcal{F}(X)=$ $X^{D}+\mathcal{F}_{1} X^{D-1}+\cdots+\mathcal{F}_{D}$, applying Proposition 3.1, and using $d_{0} \leq d$, $D \leq d_{0}^{r} \leq d^{r}$, we infer that

$$
g_{M_{i}} \leq \Delta_{i} D \max _{1 \leq k \leq D} \operatorname{deg}_{z_{i}} \mathcal{F}_{k} \leq \Delta_{i} D\left(2 d_{0}\right)^{\exp O(r)} \leq \Delta_{i}(n d)^{\exp O(r)} .
$$

Lastly, we estimate $\left|S_{i}\right|$. Each valuation of $\overline{\mathbb{k}}_{i}\left(z_{i}\right)$ can be extended to at most $\left[M_{i}: \overline{\mathbb{k}}_{i}\left(z_{i}\right)\right]=\Delta_{i}$ valuations of $M_{i}$. Thus $M_{i}$ has at most $\Delta_{i}$ valuations $v$ with $v\left(z_{i}\right)<0$ and at most $\Delta_{i} \operatorname{deg} f$ valuations $v$ with $v(f)>0$. Hence using Proposition 3.3, we get

$$
\left|S_{i}\right| \leq \Delta_{i}+\Delta_{i} \operatorname{deg}_{z_{i}} f \leq \Delta_{i}(1+\operatorname{deg} f) \leq \Delta_{i}(n d)^{\exp O(r)} .
$$

By inserting the bounds (4.17), (4.18) and (4.19) into (4.16), we infer

$$
\max \left(H_{M_{i}}\left(x^{(j)}\right), H_{M_{i}}\left(y^{(j)}\right)\right) \leq \Delta_{i}(n d)^{\exp O(r)} .
$$

In view of Lemma $4.3,(4.20), D \leq d^{r}, q \leq r$ and (4.9) we deduce that

$$
\overline{\operatorname{deg}} x, \overline{\operatorname{deg}} y \leq q D d_{1}+\sum_{i=1}^{q} \Delta_{i}^{-1} \sum_{j=1}^{D} H_{M_{i}}\left(x^{(j)}\right) \leq(n d)^{\exp O(r)} .
$$


This proves (3.16).

4.2. Hyper- and superelliptic equations. Recall the notation introduced at the beginning of Section 4 . Again, $\mathbb{k}$ is an algebraically closed field of characteristic $0, z$ a transcendental element over $\mathbb{k}, M$ a finite extension of $\mathbb{k}(z)$, and $S$ a finite subset of $\mathcal{M}_{M}$.

Proposition 4.6. Let $F \in M[X]$ be a polynomial with non-zero discriminant and $m \geq 3$ a given integer. Put $n:=\operatorname{deg} F$ and assume $n \geq 2$. All solutions of the equation

$$
F(x)=y^{m} \quad \text { in } \quad x, y \in \mathcal{O}_{S}
$$

have the property

$$
\begin{aligned}
H_{M}(x) & \leq(6 n+18) H_{M}(F)+6 g_{M / \mathbb{k}}+2|S|, \\
m H_{M}(y) & \leq\left(6 n^{2}+18 n+1\right) H_{M}(F)+6 n g_{M / \mathbb{k}}+2 n|S| .
\end{aligned}
$$

Proof. First assume that $F$ splits into linear factors over $M$, and that $S$ consists only of the infinite valuations of $M$, these are the valuations of $M$ with $v(z)<0$. Under these hypotheses, Mason [16, p.118, Theorem 15], proved that for every solution $x, y$ of (4.21) we have

$$
H_{M}(x) \leq 18 H_{M}(F)+6 g_{M / \mathbb{k}}+2(|S|-1) .
$$

But Mason's proof remains valid without any changes for any arbitrary finite set of places $S$. That is, (4.24) holds if $F$ splits into linear factors over $M$, without any condition on $S$.

We reduce the general case, where the splitting field of $M$ may be larger than $M$, to the case considered by Mason. Let $L$ be the splitting field of $F$ over $M$, and $T$ the set of valuations of $L$ that extend those of $S$. Then $|T| \leq$ $[L: M] \cdot|S|$, and by Lemma 4.2 , we have $g_{L / \mathbb{k}} \leq[L: M] \cdot\left(g_{M / \mathbb{k}}+n H_{M}(F)\right)$. Note that (4.24) holds, but with $L, T$ instead of $M, S$. It follows that

$$
\begin{aligned}
{[L: M] \cdot H_{M}(x)=H_{L}(x) } & \leq 18 H_{L}(F)+6 g_{L / \mathbb{k}}+2(|T|-1) \\
& \leq[L: M]\left((6 n+18) H_{M}(F)+6 g_{M / \mathbb{k}}+2|S|\right)
\end{aligned}
$$

which implies (4.22). Further,

$$
m H_{M}(y)=H_{M}\left(y^{m}\right)=H_{M}(F(x)) \leq H_{M}(F)+n H_{M}(x),
$$

which gives (4.23). 
Proposition 4.7. Let $F \in M[X]$ be a polynomial with non-zero discriminant. Put $n:=\operatorname{deg} F$ and assume $n \geq 3$. Then the solutions of

$$
F(x)=y^{2} \quad \text { in } \quad x, y \in \mathcal{O}_{S}
$$

have the property

$$
\begin{aligned}
& H_{M}(x) \leq(42 n+37) H_{M}(F)+8 g_{M / \mathbb{k}}+4|S|, \\
& H_{M}(y) \leq\left(21 n^{2}+19 n\right) H_{M}(F)+4 n g_{M / \mathbb{k}}+2 n|S| .
\end{aligned}
$$

Proof. First assume that $F$ splits into linear factors over $M$, that $S$ consists only of the infinite valuations of $M$, that $F$ is monic, and that $F$ has its coefficients in $\mathcal{O}_{S}$. Under these hypotheses, Mason [16, p.30, Theorem 6] proved that for every solution of (4.26) we have

$$
H_{M}(x) \leq 26 H_{M}(F)+8 g_{M / \mathbb{k}}+4(|S|-1) .
$$

An inspection of Mason's proof shows that his result is valid for arbitrary finite sets of valuations $S$, not just the set of infinite valuations. This leaves only the conditions imposed on $F$.

We reduce the general case to the special case to which (4.29) is applicable. Let $F=a_{0} X^{n}+\cdots+a_{n}$. Let $L$ be the splitting field of $F \cdot\left(X^{2}-a_{0}\right)$ over $M$. Let $T$ be the set of valuations of $L$ that extend the valuations of $S$, and also the valuations $v \in \mathcal{M}_{M}$ such that $v(F)<0$. Further, let $F^{\prime}=X^{n}+a_{1} X^{n-1}+a_{0} a_{1} X^{n-2}+\cdots+a_{0}^{n-1} a_{n}$, and let $b$ be such that $b^{2}=a_{0}^{n-1}$. Then for every solution $x, y$ of (4.26) we have

$$
F^{\prime}\left(a_{0} x\right)=(b y)^{2}, \quad a_{0} x, b y \in \mathcal{O}_{T},
$$

and moreover, $F^{\prime} \in \mathcal{O}_{T}[X], F^{\prime}$ is monic, and $F^{\prime}$ splits into linear factors over $L$. So by (4.29),

$$
H_{L}\left(a_{0} x\right) \leq 26 H_{L}\left(F^{\prime}\right)+8 g_{L / \mathbb{k}}+4(|T|-1) .
$$

First notice that

$$
H_{L}\left(F^{\prime}\right)=[L: M] H_{M}\left(F^{\prime}\right) \leq[L: M] \cdot n H_{M}(F) .
$$

Further,

$$
|T| \leq[L: M]\left(|S|-\sum_{v \in \mathcal{M}_{M}} \min (0, v(F))\right) \leq[L: M]\left(|S|+H_{M}(F)\right) .
$$

Finally, by $H_{M}\left(F \cdot\left(X^{2}-a_{0}\right)\right) \leq 2 H_{M}(F)$ and Lemma 4.2, we have

$$
g_{L / \mathbb{k}} \leq[L: M]\left(g_{M / \mathbb{k}}+(n+2) 2 H_{M}(F)\right) .
$$


By inserting these bounds into (4.30), we infer

$$
\begin{aligned}
{[L: M] H_{M}(x) } & \leq[L: M]\left(H_{M}\left(a_{0} x\right)+H_{M}(F)\right)=H_{L}\left(a_{0} x\right)+[L: M] H_{M}(F) \\
& \leq[L: M]\left((42 n+37) H_{M}(F)+8 g_{M / \mathbb{k}}+4|S|\right) .
\end{aligned}
$$

This implies (4.27). The other inequality (4.28) follows by combining (4.27) with (4.25) with $m=2$.

The final step of this subsection is to prove statement (3.19) in Proposition 3.7 .

Proof of (3.19). We closely follow the proof of statement (3.16) in Proposition 3.6, and use the same notation. In particular, $\mathbb{k}_{i}, M_{i}, S_{i}, \Delta_{i}$ will have the same meaning, and for $\alpha \in B, j=1, \ldots, D$, the $j$-th conjugate $\alpha^{(j)}$ is the one corresponding to $w^{(j)}$. Put $F^{\prime}:=\delta^{-1} F$, and let $F^{\prime(j)}$ be the polynomial obtained by taking the $j$-th conjugates of the coefficients of $F^{\prime}$.

We keep the argument together for both hyper- and superelliptic equations by using the worse bounds everywhere. Let $x, y \in B$ be a solution of (2.4), where $m, n \geq 2$ and $n \geq 3$ if $m=2$. Then

$$
F^{\prime(j)}\left(x^{(j)}\right)=\left(y^{(j)}\right)^{m}, \quad x^{(j)}, y^{(j)} \in \mathcal{O}_{S_{i}} .
$$

By combining Propositions 4.6 and 4.7 we obtain the generous bound

$$
H_{M_{i}}\left(x^{(j)}\right), m H_{M_{i}}\left(y^{(j)}\right) \leq 80 n^{2}\left(H_{M_{i}}\left(F^{\prime(j)}\right)+g_{M_{i} / \mathbb{k}_{i}}+\left|S_{i}\right|\right) .
$$

For $H_{M_{i}}\left(F^{\prime(j)}\right), g_{M_{i} / \mathbb{k}_{i}},\left|S_{i}\right|$ we have precisely the same estimates as (4.17), (4.18), (4.19). Then a similar computation as in the proof of (3.16) leads to

$$
H_{M_{i}}\left(x^{(j)}\right), m H_{M_{i}}\left(y^{(j)}\right) \leq \Delta_{i}(n d)^{\exp O(r)} .
$$

Now employing Lemma 4.3 and ignoring for the moment $m$ we get similarly as in the proof of (3.16),

$$
\overline{\operatorname{deg}} x, \overline{\operatorname{deg}} y \leq(n d)^{\exp O(r)} .
$$

It remains to estimate $m \overline{\operatorname{deg}} y$. If $y \in \overline{\mathbb{Q}}$ we have $\overline{\operatorname{deg}} y=0$. Assume that $y \notin \overline{\mathbb{Q}}$. Then $y \notin \mathbb{k}_{i}$ for at least one index $i$. Since $y \in B \subset \mathbb{k}_{i}\left(z_{i}, w\right)$ and $\left[\mathbb{k}_{i}\left(z_{i}, w\right): \mathbb{k}_{i}\left(z_{i}\right)\right] \leq D$, we have

$$
H_{M_{i}}(y)=\left[M_{i}: \mathbb{k}_{i}\left(z_{i}, w\right)\right] H_{\mathbb{k}_{i}\left(z_{i}, w\right)}(y) \geq\left[M_{i}: \mathbb{k}_{i}\left(z_{i}, w\right)\right] \geq \Delta_{i} / D .
$$


Together with (4.31) and $D \leq d^{r}$ this implies

$$
m \leq(n d)^{\exp O(r)} .
$$

This concludes the proof of (3.19).

\section{Specializations}

In this section we shall consider specialization homomorphisms from the domain $B$ to $\overline{\mathbb{Q}}$, and using these specializations together with earlier results concerning our equations in the number field case we shall finish the proof of Propositions 3.6 and 3.7.

We start with some notation. The set of places of $\mathbb{Q}$ is $\mathcal{M}_{\mathbb{Q}}=\{\infty\} \cup$ $\{$ primes $\}$. By $|\cdot|_{\infty}$ we denote the ordinary absolute value on $\mathbb{Q}$ and by $|\cdot|_{p}$ ( $p$ prime) the $p$-adic absolute value with $|p|_{p}=p^{-1}$. More generally, let $L$ be an algebraic number field with set of places $\mathcal{M}_{L}$. Given $v \in \mathcal{M}_{L}$, we define the absolute value $|\cdot|_{v}$ in such a way that its restriction to $\mathbb{Q}$ is $|\cdot|_{p}$ if $v$ lies above $p \in \mathcal{M}_{\mathbb{Q}}$. These absolute values satisfy the product formula

$$
\prod_{v \in \mathcal{M}_{L}}|\alpha|_{v}^{d_{v}}=1 \quad \text { for } \quad \alpha \in L^{*}
$$

where $d_{v}:=\left[L_{v}: \mathbb{Q}_{p}\right] /[L: \mathbb{Q}]$, with $p \in \mathcal{M}_{\mathbb{Q}}$ the place below $v$, and $\mathbb{Q}_{p}, L_{v}$ the completions of $\mathbb{Q}$ at $p, L$ at $v$. Note that we have $\sum_{v \mid p} d_{v}=1$ for every $p \in \mathcal{M}_{\mathbb{Q}}$. The absolute logarithmic height of $\alpha \in L$ is defined by

$$
h(\alpha):=\log \prod_{v \in \mathcal{M}_{L}} \max \left(1,|\alpha|_{v}^{d_{v}}\right) .
$$

This depends only on $\alpha$ and not on the choice of the number field $L$ containing $\alpha$, hence it defines a height on $\overline{\mathbb{Q}}$. For properties of the height we refer to Bombieri and Gubler [5].

Lemma 5.1. Let $m \geq 1$ and let $\alpha_{1}, \ldots, \alpha_{m} \in \overline{\mathbb{Q}}$ be distinct, and suppose that $G(X):=\prod_{j=1}^{m}\left(X-\alpha_{j}\right) \in \mathbb{Z}[X]$. Let $q, p_{0}, \ldots, p_{m-1}$ be integers with $\operatorname{gcd}\left(q, p_{0}, \ldots, p_{m-1}\right)=1$ and $p u t$

$$
\beta_{j}:=\sum_{i=0}^{m-1} \frac{p_{j}}{q} \alpha_{j}^{i}, \quad j=1, \ldots, m .
$$


Then

$$
\log \max \left(|q|,\left|p_{0}\right|, \ldots,\left|p_{m-1}\right|\right) \leq 2 m^{2}+(m-1) h(G)+\sum_{j=1}^{m} h\left(\beta_{j}\right) .
$$

Proof. This is Lemma 5.2 in Evertse and Györy [9].

We now consider our specializations $B \mapsto \overline{\mathbb{Q}}$ and prove some of their properties. These specializations were introduced by Győry [11] and [12] and, in a refined form, by Evertse and Györy [9].

We assume $q>0$ and apart from that keep the notation and assumption from Section 3. In particular, $K_{0}:=\mathbb{Q}\left(z_{1}, \ldots, z_{q}\right), K:=\mathbb{Q}\left(z_{1}, \ldots, z_{q}, w\right)$, $A_{0}:=\mathbb{Z}\left[z_{1}, \ldots, z_{q}\right]$. Further, $B:=\mathbb{Z}\left[z_{1}, \ldots, z_{q}, f^{-1}, w\right]$ where $f$ is a nonzero element of $A_{0}$ with the properties specified in Proposition 3.3, and $w$ is integral over $A_{0}$ and has minimal polynomial

$$
\mathcal{F}(X)=X^{D}+\mathcal{F}_{1} X^{D-1}+\cdots+\mathcal{F}_{D} \in A_{0}[X]
$$

over $K_{0}$ as in Proposition 3.1 (i). In the case $D=1$ we take $w=1$, $\mathcal{F}(X)=X-1$.

Let $\mathbf{u}=\left(u_{1}, \ldots, u_{q}\right) \in \mathbb{Z}^{q}$. Then the substitution $z_{1} \rightarrow u_{1}, \ldots, z_{q} \rightarrow u_{q}$ defines a ring homomorphism (specialization) from $K_{0}$ to $\mathbb{Q}$

$$
\varphi_{\mathbf{u}}: \alpha \mapsto \alpha(\mathbf{u}):\left\{\alpha=\frac{g_{1}}{g_{2}}: g_{1}, g_{2} \in A_{0}, g_{2}(\mathbf{u}) \neq 0\right\} \rightarrow \mathbb{Q} .
$$

To extend this to a ring homomorphism from $B$ to $\overline{\mathbb{Q}}$ we have to impose some restrictions on $\mathbf{u}$. Let $\Delta_{\mathcal{F}}$ be the discriminant of $\mathcal{F}$ (with $\Delta_{\mathcal{F}}=1$ if $D=1$ ), and let

$$
\mathcal{H}:=\Delta_{\mathcal{F}} \cdot \mathcal{F}_{D} \cdot f
$$

Put

$$
\left\{\begin{array}{l}
d_{0}^{*}:=\max \left(\operatorname{deg} \mathcal{F}_{1}, \ldots, \operatorname{deg} \mathcal{F}_{D}\right), \quad d_{1}^{*}:=\max \left(d_{0}^{*}, \operatorname{deg} f\right) \\
h_{0}^{*}:=\max \left(h\left(\mathcal{F}_{1}\right), \ldots, h\left(\mathcal{F}_{D}\right)\right), \quad h_{1}^{*}:=\max \left(h_{0}^{*}, h(f)\right) .
\end{array}\right.
$$

Clearly $\mathcal{H} \in A_{0}$ and since $\Delta_{\mathcal{F}}$ is a homogeneous polynomial in $\mathcal{F}_{1}, \ldots, \mathcal{F}_{D}$ of degree $2 D-2$, we have

$$
\operatorname{deg} \mathcal{H} \leq(2 D-1) d_{0}^{*}+d_{1}^{*} .
$$


Further, by Proposition 3.1 (i), Proposition 3.3 and (2.2) we also have

$$
\begin{cases}d_{0}^{*} \leq(2 d)^{\exp O(r)}, & h_{0}^{*} \leq(2 d)^{\exp O(r)}(h+1), \\ d_{1}^{*} \leq(n d)^{\exp O(r)}, & h_{1}^{*} \leq(n d)^{\exp O(r)}(h+1)\end{cases}
$$

Next assume that

$$
\mathcal{H}(\mathbf{u}) \neq 0 \text {. }
$$

Then we have $f(\mathbf{u}) \neq 0, \Delta_{F}(\mathbf{u}) \neq 0$, hence the polynomial

$$
\mathcal{F}_{\mathbf{u}}:=X^{D}+\mathcal{F}_{1}(\mathbf{u}) X^{D-1}+\cdots+\mathcal{F}_{D}(\mathbf{u})
$$

has $D$ distinct zeros which are all different from 0 , say $w^{(1)}(\mathbf{u}), \ldots, w^{(D)}(\mathbf{u})$. Consequently, for $j=1, \ldots, D$ the assignment

$$
z_{1} \mapsto u_{1}, \ldots, z_{q} \mapsto u_{q}, w \mapsto w^{(j)}(\mathbf{u})
$$

defines a ring homomorphism $\varphi_{\mathbf{u}, j}$ from $B$ to $\overline{\mathbb{Q}}$; if $D=1$ it is just $\varphi_{\mathbf{u}}$. The image of $\alpha \in B$ under $\varphi_{\mathbf{u}, j}$ is denoted by $\alpha^{(j)}(\mathbf{u})$. It is important to note that if $\alpha$ is a unit in $B$, then its image by a specialization cannot be 0 . Thus by Proposition $3.3, \delta(\mathbf{u}) \neq 0$ and $D_{F}(\mathbf{u}) \neq 0$.

Recall that we may express elements of $B$ as

$$
\begin{aligned}
\alpha= & \sum_{i=1}^{D-1}\left(P_{i} / Q\right) w^{i} \\
& \quad \text { where } P_{0}, \ldots, P_{D-1}, Q \in A_{0}, \operatorname{gcd}\left(P_{0}, \ldots, P_{D-1}, Q\right)=1 .
\end{aligned}
$$

Because of $\alpha \in B, Q$ must divide a power of $f$; hence $Q(\mathbf{u}) \neq 0$. So we have

$$
\alpha^{(j)}(\mathbf{u})=\sum_{i=1}^{D-1}\left(P_{i}(\mathbf{u}) / Q(\mathbf{u})\right)\left(w^{(j)}(\mathbf{u})\right)^{i}, \quad j=1, \ldots, D .
$$

Clearly, $\varphi_{\mathbf{u}, j}$ is the identity on $B \cap \mathbb{Q}$. Hence if $\alpha \in B \cap \overline{\mathbb{Q}}$ then $\varphi_{\mathbf{u}, j}(\alpha)$ has the same minimal polynomial as $\alpha$ and so it is a conjugate of $\alpha$.

For $\mathbf{u}=\left(u_{1}, \ldots, u_{q}\right) \in \mathbb{Z}^{q}$, put $|\mathbf{u}|:=\max \left(\left|u_{1}\right|, \ldots,\left|u_{q}\right|\right)$. It is easy to check that for any $g \in A_{0}, \mathbf{u} \in \mathbb{Z}^{q}$

$$
\log |g(\mathbf{u})| \leq q \log \operatorname{deg} g+h(g)+\operatorname{deg} g \log \max (1,|\mathbf{u}|) .
$$

In particular, we have

$$
h\left(\mathcal{F}_{\mathbf{u}}\right) \leq q \log d_{0}^{*}+h_{0}^{*}+d_{0}^{*} \log \max (1,|\mathbf{u}|)
$$


and so by Lemma 5.1 of Evertse and Győry [9]

$$
\sum_{j=1}^{D} h\left(w^{(j)}(\mathbf{u})\right) \leq D+1+q \log d_{0}^{*}+h_{0}^{*}+d_{0}^{*} \log \max (1,|\mathbf{u}|) .
$$

We define the algebraic number fields $K_{\mathbf{u}, j}=\mathbb{Q}\left(w^{(j)}(\mathbf{u})\right)$ for $j=1, \ldots, D$. We denote by $\Delta_{L}$ the the discriminant of an algebraic number field $L$. We derive an upper bound for the absolute value of the discriminant $\Delta_{K_{\mathbf{u}, j}}$ of $K_{\mathbf{u}, j}$.

Lemma 5.2. Let $\mathbf{u} \in \mathbb{Z}^{q}$ with $\mathcal{H}(\mathbf{u}) \neq 0$. Then for $j=1, \ldots, D$ we have $\left[K_{\mathbf{u}, j}: \mathbb{Q}\right] \leq D$ and

$$
\left|\Delta_{K_{\mathbf{u}, j}}\right| \leq D^{2 D-1}\left(\left(d_{0}^{*}\right)^{q} e^{h_{0}^{*}} \max \left(1,|\mathbf{u}|^{d_{0}^{*}}\right)\right)^{2 D-2} .
$$

Proof. This is Lemma 5.5 in Evertse and Győry [9].

The following two lemmas relate the height of $\alpha \in B$ to the heights of $\alpha^{(j)}(\mathbf{u})$ for $\mathbf{u} \in \mathbb{Z}^{q}$.

Lemma 5.3. Let $\mathbf{u} \in \mathbb{Z}^{q}$ with $\mathcal{H}(\mathbf{u}) \neq 0$, and let $\alpha \in B$. Then for $j=$ $1, \ldots, D$,

$$
\begin{aligned}
h\left(\alpha^{(j)}(\mathbf{u})\right) \leq & D^{2}+q\left(D \log d_{0}^{*}+\log \overline{\operatorname{deg}} \alpha\right)+ \\
& +D h_{0}^{*}+\bar{h}(\alpha)+\left(D d_{0}^{*}+\overline{\operatorname{deg}} \alpha\right) \log \max (1,|\mathbf{u}|) .
\end{aligned}
$$

Proof. This is Lemma 5.6 in Evertse and Győry [9].

Lemma 5.4. Let $\alpha \in B, \alpha \neq 0$, and let $N$ be an integer with

$$
N \geq \max \left(\overline{\operatorname{deg}} \alpha, 2 D d_{0}^{*}+2(q+1)\left(d_{1}^{*}+1\right)\right) .
$$

Then the set

$$
\mathcal{S}:=\left\{\mathbf{u} \in \mathbb{Z}^{q}:|\mathbf{u}| \leq N, \mathcal{H}(\mathbf{u}) \neq 0\right\}
$$

is non-empty, and

$$
\bar{h}(\alpha) \leq 5 N^{4}\left(h_{1}^{*}+1\right)^{2}+2 D\left(h_{1}^{*}+1\right) H,
$$

where $H:=\max \left\{h\left(\alpha^{(j)}(\mathbf{u})\right): \mathbf{u} \in \mathcal{S}, j=1, \ldots, D\right\}$.

Proof. This is Lemma 5.7 in Evertse and Győry [9]. 


\section{Bounding The HeIGHT AND THE EXPONENT $m$}

We shall derive the height bounds (3.17) in Proposition 3.6 and (3.20) in Proposition 3.7, as well as the upper bound for $m$ in Proposition 3.8 by combining the specialization techniques from the previous section with existing effective results for Diophantine equations over $S$-integers of a number field, namely Győry and $\mathrm{Yu}$ [13] for Thue equations, and the three authors [4] for hyper- and superelliptic equations and the Schinzel-Tijdeman equation.

6.1. Thue equations. In the statement of the result of Györy and Yu we need some notation.

For an algebraic number field $L$, we denote by $d_{L}, \mathcal{O}_{L}, \mathcal{M}_{L}, \Delta_{L}, h_{L}$, $r_{L}$ and $R_{L}$ the degree, ring of integers, set of places, discriminant, class number, unit rank and regulator of $L$. The absolute norm of an ideal $\mathfrak{a}$ of $\mathcal{O}_{L}$ is denoted by $N(\mathfrak{a})$.

Let $L$ be an algebraic number field and let $S$ be a finite set of places of $L$ which contains all infinite places. Denote by $s$ the cardinality of $S$. Recall that the ring of $S$-integers $\mathcal{O}_{S}$ is defined as

$$
\mathcal{O}_{S}=\left\{\alpha \in L:|\alpha|_{v} \leq 1 \text { for } v \in \mathcal{M}_{L} \backslash S\right\} .
$$

If $S$ consists only of the infinite places of $L$, we put $P:=2, Q:=2$. If $S$ contains also finite places, we denote by $\mathfrak{p}_{1}, \ldots, \mathfrak{p}_{t}$ the prime ideals corresponding to the finite places of $S$, and we put

$$
P:=\max \left(N\left(\mathfrak{p}_{1}\right), \ldots, N\left(\mathfrak{p}_{t}\right)\right), \quad Q:=N\left(\mathfrak{p}_{1} \ldots \mathfrak{p}_{t}\right) .
$$

The $S$-regulator associated with $S$ is denoted by $R_{S}$. If $S$ consists only of the infinite places of $L$ it is just $R_{L}$, while otherwise

$$
R_{S}=h_{S} R_{L} \prod_{i=1}^{t} \log N\left(\mathfrak{p}_{i}\right),
$$

where $h_{S}$ is a (positive) divisor of $h_{L}$. It is an easy consequence of formula (2) of Louboutin [15] that

$$
h_{L} R_{L} \leq\left|\Delta_{L}\right|^{1 / 2}\left(\log ^{*}\left|\Delta_{L}\right|\right)^{d_{L}-1} ;
$$

cf. formula (59) of Győry and Yu, [13]. Further, we have

$$
R_{S} \leq\left|\Delta_{L}\right|^{1 / 2}\left(\log ^{*}\left|\Delta_{L}\right|\right)^{d_{L}-1}\left(\log ^{*} Q\right)^{s} ;
$$

see (6.1) in Evertse and Győry [9]. In view of (6.1) this is true also if $t=0$. 
6.1.1. Results in the number field case. Let $F(X, Y) \in L[X, Y]$ be a binary form of degree $n \geq 3$ with splitting field $L$ and with at least three pairwise non-proportional linear factors. Further, let $\beta \in L \backslash\{0\}$ and consider the Thue equation

$$
F(\xi, \eta)=\beta \quad \text { in } \quad \xi, \eta \in \mathcal{O}_{S} .
$$

For a polynomial $G$ with algebraic coefficients, we denote by $h(G)$ the maximum of the logarithmic heights of its coefficients.

Proposition 6.1. All solutions $(\xi, \eta) \in \mathcal{O}_{S}^{2}$ of equation (6.3) satisfy

$$
\begin{aligned}
\max (h(\xi), h(\eta)) \leq c_{1} P R_{S}\left(1+\left(\log ^{*} R_{S}\right) / \log ^{*} P\right) \times \\
\times\left(c_{2} R_{L}+\frac{h_{L}}{d_{L}} \log Q+2 n d_{L} H_{1}+H_{2}\right),
\end{aligned}
$$

where

$$
\begin{gathered}
H_{1}=\max (1, h(F)), \quad H_{2}=\max (1, h(\delta)), \\
c_{1}=250 n^{6} s^{2 s+3.5} \cdot 2^{7 s+27}(\log 2 s) d_{L}^{2 s+4}\left(\log ^{*}\left(2 d_{L}\right)\right)^{3}
\end{gathered}
$$

and

$$
c_{2}= \begin{cases}0 & \text { if } r_{L}=0 \\ 1 / d_{L} & \text { if } r_{L}=1 \\ 29 e r_{L} ! r_{L} \sqrt{r_{L}-1} \log d_{L} & \text { if } r_{L} \geq 2 .\end{cases}
$$

Proof. This is Corollary 3 of Győry and Yu [13].

We shall also need the following.

Lemma 6.2. If $L$ is the composite of the algebraic number fields $L_{1}, \ldots, L_{k}$ with degrees $d_{L_{1}}, \ldots, d_{L_{k}}$ and discriminants $\Delta_{L_{1}}, \ldots, \Delta_{L_{k}}$, then $\Delta_{L}$ divides $\Delta_{L_{1}}^{d_{L} / d_{L_{1}}} \ldots \Delta_{L_{k}}^{d_{L} / d_{L_{k}}}$ in $\mathbb{Z}$

Proof. See Stark [20].

Lemma 6.3. Let $L$ be an algebraic number field and $\theta$ a zero of a polynomial $G \in L[X]$ of degree $n$ without multiple roots. Then

$$
\left|\Delta_{L(\theta)}\right| \leq n^{(2 n-1) d_{L}} e^{\left(2 n^{2}-2\right) h(G)}\left|\Delta_{L}\right|^{[L(\theta): L]} .
$$


Proof. This is a slight modification of the second assertion of [4, Lemma 4.1]. In fact, this lemma gives the same bound but with an exponent $(2 n-2) h^{\prime}(G)$ on $e$, where for $G=\sum_{k=0}^{n} b_{k} X^{n-k}$ we define

$$
h^{\prime}(G)=\sum_{v \in \mathcal{M}_{L}} d_{v} \log \max \left(1,\left|b_{0}\right|_{v}, \ldots,\left|b_{n}\right|_{v}\right) .
$$

This height is easily estimated from above by $\sum_{k=0}^{n} h\left(b_{k}\right) \leq(n+1) h(G)$. Our lemma follows.

\subsubsection{Concluding the proof of Proposition 3.6.}

Proof of (3.17) in Proposition 3.6. We first consider the case $q>0$. Let $x, y$ be a solution of (3.15) in $B$. We keep the notation introduced in Section 5 . Recall that $\mathcal{H}:=\Delta_{\mathcal{F}} \cdot \mathcal{F}_{D} \cdot f$ and by (5.3) and (5.4) we get

$$
\operatorname{deg} \mathcal{H} \leq(n d)^{\exp O(r)} \text {. }
$$

Choose $\mathbf{u} \in \mathbb{Z}^{q}$ with $\mathcal{H}(\mathbf{u}) \neq 0$, choose $j \in\{1, \ldots, D\}$, and denote by $F_{\mathbf{u}, j}, \delta^{(j)}(\mathbf{u}), x^{(j)}(\mathbf{u}), y^{(j)}(\mathbf{u})$, the images of $F, \delta, x, y$ under $\varphi_{\mathbf{u}, j}$. Then $F_{\mathbf{u}, j}$ has its coefficients in $K_{\mathbf{u}, j}$. Further, let $L$ denote the splitting field of $F_{\mathbf{u}, j}$ over $K_{\mathbf{u}, j}$, and $S$ the set of places of $L$ which consists of all infinite places and all finite places lying above the rational prime divisors of $f(u)$. Note that $w^{(j)}(\mathbf{u})$ is an algebraic integer and $f(\mathbf{u}) \in \mathcal{O}_{S}^{*}$. Thus $\varphi_{\mathbf{u}, j}(B) \subseteq \mathcal{O}_{S}$ and it follows from (3.15) that

$$
F_{\mathbf{u}, j}\left(x^{(j)}(\mathbf{u}), y^{(j)}(\mathbf{u})\right)=\delta^{(j)}(\mathbf{u}), \quad x^{(j)}(\mathbf{u}), y^{(j)}(\mathbf{u}) \in \mathcal{O}_{S} .
$$

We already proved in Section 4 that (3.16) of Proposition 3.6 holds, i.e. we have

$$
\overline{\operatorname{deg}} x, \overline{\operatorname{deg}} y \leq(n d)^{\exp O(r)} .
$$

Hence we can apply Lemma 5.4 with

$$
N=\max \left((n d)^{\exp O(r)}, 2 D d_{0}^{*}+2(q+1)\left(d_{1}^{*}+1\right)\right) .
$$

In view of (5.4), D $\leq d^{r}$ and $q \leq r$ we get

$$
N \leq(n d)^{\exp O(r)} \text {. }
$$

By applying Lemma 5.4 with $\alpha=x$ and $\alpha=y$, and inserting $D \leq d^{r}$ and the upper bound $h_{1}^{*} \leq(n d)^{\exp O(r)}(h+1)$ from (5.4), it follows that there are $\mathbf{u} \in \mathbb{Z}^{q}, j \in\{1, \ldots, D\}$ with

$$
|\mathbf{u}| \leq(n d)^{\exp O(r)}, \quad \mathcal{H}(\mathbf{u}) \neq 0
$$


and

$$
\begin{aligned}
\max (\bar{h}(x), \bar{h}(y)) \leq(n d)^{\exp O(r)}\left[(h+1)^{2}+\right. & \\
+ & \left.d^{r}(h+1) \max \left(h\left(x^{(j)}(\mathbf{u})\right), h\left(y^{(j)}(\mathbf{u})\right)\right)\right] .
\end{aligned}
$$

We proceed further with this $\mathbf{u}, j$ and apply Proposition 6.1 to equation (6.6) to derive an upper bound for $h\left(x^{(j)}(\mathbf{u})\right)$ and $h\left(y^{(j)}(\mathbf{u})\right)$. To do so we have to bound from above the parameters corresponding to those which occur in Proposition 6.1.

Write $F=\sum_{k=0}^{n} a_{k} X^{n-k} Y^{k}$ and put

$$
\overline{\operatorname{deg}} F:=\max _{0 \leq k \leq n} \overline{\operatorname{deg}} a_{k}, \quad \bar{h}(F):=\max _{0 \leq k \leq n} \bar{h}\left(a_{k}\right) .
$$

Notice that by Lemma 3.4, applied to $\delta$ and the coefficients of $F$ with the choice $d^{*}=d, h^{*}=h$, we have

$$
\begin{aligned}
\overline{\operatorname{deg}} F, \overline{\operatorname{deg}} \delta & \leq(2 d)^{\exp O(r)}, \\
\bar{h}(F), \bar{h}(\delta) & \leq(2 d)^{\exp O(r)}(h+1) .
\end{aligned}
$$

It follows from Lemma $5.3, q \leq r, D \leq d^{r},(5.4),(6.10),(6.11)$, and lastly (6.8), that

$$
\begin{aligned}
h\left(F_{\mathbf{u}, j}\right) \leq & D^{2}+q\left(D \log d_{0}^{*}+\log \overline{\operatorname{deg}} F\right)+D h_{0}^{*}+ \\
& +\bar{h}(F)+\left(D d_{0}^{*}+\overline{\operatorname{deg}} F\right) \log \max (1,|\mathbf{u}|) \\
\leq & (n d)^{\exp O(r)}(h+1) .
\end{aligned}
$$

In a similar way, replacing $F$ by $\delta$, we obtain also

$$
h\left(\delta^{(j)}(\mathbf{u})\right) \leq(n d)^{\exp O(r)}(h+1) .
$$

We recall that $d_{L}$ and $\Delta_{L}$ denote the degree and the discriminant of $L$ over $\mathbb{Q}$. Since $\left[K_{\mathbf{u}, j}: \mathbb{Q}\right] \leq D$, we have $d_{L} \leq D n$ !. Let $G(X):=F(X, 1)$, and let $\theta_{1}, \ldots, \theta_{n^{\prime}}$ be the roots of $G$. We have $n^{\prime}=n$ if $a_{0} \neq 0$ and $n^{\prime}=n-1$ otherwise. Then $L=K_{\mathbf{u}, j}\left(\theta_{1}, \ldots, \theta_{n^{\prime}}\right)$. Denote by $d_{L_{i}}$ the degree and by $\Delta_{L_{i}}$ the discriminant of the number field $L_{i}:=K_{\mathbf{u}, j}\left(\theta_{i}\right), i=1, \ldots, n^{\prime}$. Then by Lemma 6.2 we have

$$
\left|\Delta_{L}\right| \leq \prod_{i=1}^{n^{\prime}}\left|\Delta_{L_{i}}\right|^{d_{L} / d_{L_{i}}}
$$


We estimate $\left|\Delta_{L}\right|$. First notice that by Lemma 5.2, inserting the estimates $q \leq r, D \leq d^{r},(5.4),(6.8)$,

$$
\begin{aligned}
\left|\Delta_{K_{\mathbf{u}, j}}\right| & \leq D^{2 D-1}\left(\left(d_{0}^{*}\right)^{q} e^{h_{0}^{*}} \max \left(1,|\mathbf{u}|^{d_{0}^{*}} \mid\right)\right)^{2 D-2} \\
& \leq \exp \left((n d)^{\exp O(r)}(h+1)\right) .
\end{aligned}
$$

Further, by Lemma 6.3 and the estimates $D \leq d^{r}$, (6.12), (6.15),

$$
\begin{aligned}
\left|\Delta_{L_{i}}\right| & \leq n^{(2 n-1) D} e^{\left(2 n^{2}-2\right) h\left(F_{\mathbf{u}, j}\right)}\left|\Delta_{K_{\mathbf{u}, j}}\right|{ }^{\left[L_{i}: K_{\mathbf{u}, j}\right]} \\
& \leq \exp \left\{\left[L_{i}: K_{\mathbf{u}, j}\right] \cdot(n d)^{\exp O(r)}(h+1)\right\} .
\end{aligned}
$$

By inserting this into (6.14), using $\left[L: K_{\mathbf{u}, j}\right] \leq n$ !, we obtain

$$
\begin{aligned}
\left|\Delta_{L}\right| & \leq \exp \left\{(n d)^{\exp O(r)}(h+1) \cdot n d_{L} / d_{K_{\mathbf{u}, j}}\right\} \\
& \leq \exp \left\{n !(n d)^{\exp O(r)}(h+1)\right\} .
\end{aligned}
$$

By assumption (5.2), $f$ has degree at most $d_{1}^{*}$ and logarithmic height at most $h_{1}^{*}$. Further, $f(\mathbf{u}) \neq 0$ and by $q \leq r,(5.4)$, (6.8),

$$
|f(\mathbf{u})| \leq\left(d_{1}^{*}\right)^{q} e^{h_{1}^{*}} \max (1,|\mathbf{u}|)^{d_{1}^{*}} \leq \exp \left\{(n d)^{\exp O(r)}(h+1)\right\} .
$$

The cardinality $s$ of $S$ is at most $d_{L}(1+\omega)$, where $\omega$ denotes the number of distinct prime divisors of $f(\mathbf{u})$. By prime number theory,

$$
s=O\left(d_{L} \log ^{*}|f(\mathbf{u})| / \log ^{*} \log ^{*}|f(\mathbf{u})|\right) .
$$

From this estimate and (6.17), $D \leq d^{r}, d_{L} \leq n ! d^{r}$, one easily deduces that for $c_{1}$ coming from Proposition 6.1 we have

$$
c_{1} \leq \exp \left\{n !(n d)^{\exp O(r)}(h+1)\right\} .
$$

Next, we estimate $P, Q$ and $R_{S}$. By $(6.17), d_{L} \leq n ! d^{r}$ we have

$$
P \leq Q \leq|f(\mathbf{u})|^{d_{L}} \leq \exp \left\{n !(n d)^{\exp O(r)}(h+1)\right\} .
$$

To estimate $R_{S}$, we use (6.2). Then, in view of (6.16) and $d_{L} \leq n ! d^{r}$, we have

$$
\left|\Delta_{L}\right|^{1 / 2}\left(\log ^{*}\left|\Delta_{L}\right|\right)^{d_{L}-1} \leq \exp \left\{n !(n d)^{\exp O(r)}(h+1)\right\} .
$$

Further, by (6.18) and (6.20),

$$
(\log Q)^{s} \leq \exp \left\{O\left(d_{L} \frac{\log ^{*}|f(\mathbf{u})|}{\log ^{*} \log ^{*}|f(\mathbf{u})|} \cdot\left(\log d_{L}+\log ^{*} \log ^{*}|f(\mathbf{u})|\right)\right)\right\} .
$$

Together with (6.17), this leads to

$$
R_{S} \leq\left|\Delta_{L}\right|^{1 / 2}\left(\log ^{*}\left|\Delta_{L}\right|\right)^{d_{L}-1}(\log Q)^{s} \leq \exp \left\{n !(n d)^{\exp O(r)}(h+1)\right\} .
$$


Combining (6.1) with (6.21) and with $R_{L}>0.2052$ (see Friedman [10]) we get

$$
\max \left(h_{L}, R_{L}\right) \leq \exp \left\{n !(n d)^{\exp O(r)}(h+1)\right\}
$$

Finally, using $r_{L}<d_{L} \leq n ! d^{r}$, we infer that

$$
c_{2} \leq \exp O\left(d_{L} \log ^{*} d_{L}\right) \leq \exp \left\{n !(n d)^{\exp O(r)}\right\} .
$$

We now apply Proposition 6.1 to equation (6.6). From the estimates (6.12), (6.13), (6.19), (6.20), (6.22), (6.23), (6.24), it follows that the upper bound in Proposition 6.1 is a sum and product of terms, which are all bounded above by $\exp \left\{n !(n d)^{\exp O(r)}(h+1)\right\}$. It follows that

$$
h\left(x^{(j)}(\mathbf{u})\right), h\left(y^{(j)}(\mathbf{u})\right) \leq \exp \left\{n !(n d)^{\exp O(r)}(h+1)\right\} .
$$

By inserting this into (6.9), we obtain the upper bound (3.17) in Proposition 3.6 for $q>0$.

Now assume $q=0$. In this case $K_{0}=\mathbb{Q}, A_{0}=\mathbb{Z}$ and $B=\mathbb{Z}\left[f^{-1}, w\right]$, where $w$ is an algebraic integer with minimal polynomial $\mathcal{F}(X)=X^{D}+$ $\mathcal{F}_{1} X^{D-1}+\cdots+\mathcal{F}_{D} \in \mathbb{Z}[X]$ over $\mathbb{Q}$, and $f$ is a non-zero rational integer. In view of Propositions 3.1 (i) and 3.3 we may assume that

$$
\log |f| \leq h_{1}^{*} \quad \text { and } \quad \log \left|\mathcal{F}_{k}\right| \leq h_{0}^{*} \quad \text { for } \quad k=1, \ldots, D,
$$

where $h_{0}^{*}, h_{1}^{*}$ satisfy (5.4). Denote by $w^{(1)}, \ldots, w^{(D)}$ the conjugates of $w$, and let $K_{j}:=\mathbb{Q}\left(w^{(j)}\right)$ for $j:=1, \ldots, D$. By a similar argument as in the proof of Lemma 5.5 of Evertse and Györy [9], we have $\left|\Delta_{K_{j}}\right| \leq D^{2 D-1} e^{(2 D-2) h_{0}^{*}}$, which is the estimate from Lemma 5.2 with $q=0$ and $\max (1,|\mathbf{u}|)$ replaced by 1 . For $\alpha \in K$, we denote by $\alpha^{(j)}$ the conjugate of $\alpha$ corresponding to $w^{(j)}$.

Instead of Lemma 5.4 we use Lemma 5.1, applied with $G=\mathcal{F}, m=D$ and $\beta^{(j)}=x^{(j)}$, resp. $y^{(j)}$. Inserting (5.4), this leads to an estimate

$$
\max (\bar{h}(x), \bar{h}(y)) \leq(n d)^{\exp O(r)} \max _{1 \leq j \leq D} \max \left(h\left(x^{(j)}\right), h\left(y^{(j)}\right)\right) .
$$

We proceed further with the $j$ for which the maximum is assumed.

Now we can follow the argument for the case $q>0$, except that in all estimates we have to take $q=0$, and replace $\max (1,|\mathbf{u}|)$ by $1, K_{\mathbf{u}, j}$ by $K_{j}$, $f(\mathbf{u})$ by $f, F_{\mathbf{u}, j}$ by $F^{(j)}$, where $F^{(j)}$ is the binary form obtained by taking 
the $j$-th conjugates of the coefficients of $F$, and $f(\mathbf{u})$ by $f$. This leads to an estimate

$$
h\left(\left(x^{(j)}\right)\right), h\left(\left(y^{(j)}\right) \leq \exp \left\{n !(n d)^{\exp O(r)}(h+1)\right\},\right.
$$

and combined with (6.25) this gives again (3.17). This completes the proof of Proposition 3.6.

\subsection{Hyper- and superelliptic equations.}

6.2.1. Results in the number field case. Let $L$ be a number field, and denote as usual by $d_{L}, \Delta_{L}, \mathcal{O}_{L}, \mathcal{M}_{L}$ its degree, discriminant, class number, regulator, ring of integers, and set of places. Further, let $S$ be a finite set of places of $L$ containing all infinite places. If $S$ consists only of the infinite places of $L$, put $P:=2, Q:=2$. Otherwise, denote by $\mathfrak{p}_{1}, \ldots, \mathfrak{p}_{t}$ the prime ideals corresponding to the finite places of $S$, and put

$$
P:=\max \left(N\left(\mathfrak{p}_{1}\right), \ldots, N\left(\mathfrak{p}_{t}\right)\right), \quad Q:=N\left(\mathfrak{p}_{1} \ldots \mathfrak{p}_{t}\right) .
$$

Let

$$
F(X)=a_{0} X^{n}+a_{1} X^{n-1}+\cdots+a_{n} \in \mathcal{O}_{S}[X]
$$

be a polynomial of degree $n \geq 2$ and of non-zero discriminant, $\delta \in \mathcal{O}_{S} \backslash\{0\}$, and $m$ a positive integer. Put

$$
\hat{h}:=\sum_{v \in \mathcal{M}_{L}} d_{v} \log \max \left(1,|\delta|_{v},\left|a_{0}\right|_{v}, \ldots,\left|a_{n}\right|_{v}\right),
$$

where $d_{v}:=\left[L_{v}: \mathbb{Q}_{p}\right] /[L: \mathbb{Q}]$, with $p \in \mathcal{M}_{\mathbb{Q}}$ the place below $v$.

Proposition 6.4. Assume $n \geq 2, m \geq 3$. If $x, y \in \mathcal{O}_{S}$ is a solution to the equation

$$
F(x)=\delta y^{m}, \quad x, y \in \mathcal{O}_{S},
$$

then

$$
h(x), h(y) \leq c_{3}^{m^{3}}\left|\Delta_{L}\right|^{2 m^{2} n^{2}} Q^{3 m^{2} n^{2}} e^{8 m^{2} n^{3} d_{L} \widehat{h}},
$$

where $c_{3}:=(6 n s)^{14 n^{3} s}$.

Proof. This is Theorem 2.1 in [4]. 
Proposition 6.5. Let $n \geq 3$. If $x, y \in \mathcal{O}_{S}$ is a solution to

$$
F(x)=\delta y^{2}, \quad x, y \in \mathcal{O}_{S},
$$

then

$$
h(x), h(y) \leq c_{4}\left|\Delta_{L}\right|^{8 n^{3}} Q^{20 n^{3}} e^{50 n^{4} d_{L} \widehat{h}},
$$

where $c_{4}:=(4 n s)^{212 n^{4} s}$.

Proof. This is Theorem 2.2 in [4].

Proposition 6.6. Let $n \geq 2$. If $x, y, m$ is a solution to

$$
F(x)=\delta y^{m}, \quad x, y \in \mathcal{O}_{S}, m \in \mathbb{Z}_{\geq 2},
$$

such that $y \neq 0$ and $y$ is not a root of unity, then

$$
m \leq c_{5}\left|\Delta_{L}\right|^{6 n} P^{n^{2}} e^{11 n d_{L} \widehat{h}}
$$

where $c_{5}:=\left(10 n^{2} s\right)^{40 n s}$.

Proof. This is Theorem 2.3 in [4].

\subsubsection{Concluding the proofs of Propositions 3.7 and 3.8.}

Proof of (3.20) in Proposition 3.7. The computations will be similar to those in the proof of (3.17) in Proposition 3.6 but with some simplifications.

First we suppose $q>0$. Take a solution $x, y$ of (3.18) in $B$. We use again the polynomial $\mathcal{H}:=\Delta_{\mathcal{F}} \cdot \mathcal{F}_{D} \cdot f$ from Section 5 . Take again $\mathbf{u} \in \mathbb{Z}^{q}$ with $\mathcal{H}(\mathbf{u}) \neq 0$, choose $j \in\{1, \ldots, D\}$, and denote by $F_{\mathbf{u}, j}, \delta^{(j)}(\mathbf{u}), x^{(j)}(\mathbf{u})$, $y^{(j)}(\mathbf{u})$, the images of $F, \delta, x, y$ under the specialization $\varphi_{\mathbf{u}, j}$. In contrast to our argument for Thue equations, we do not have to deal with the splitting field of $F$ now. So we take for $S$ the set of places of $K_{\mathbf{u}, j}$, consisting of all infinite places, and all finite places lying above the rational prime divisors of $f(\mathbf{u})$. Then $\varphi_{\mathbf{u}, j}(B) \subseteq \mathcal{O}_{S}$, and

$$
F_{\mathbf{u}, j}\left(x^{(j)}(\mathbf{u})\right)=\delta^{(j)}(\mathbf{u}) y^{(j)}(\mathbf{u})^{m}, \quad \mathbf{x}^{(j)}(\mathbf{u}), y^{(j)}(\mathbf{u}) \in \mathcal{O}_{S} .
$$

Note that by the choice of $\mathcal{H}$ and $\mathcal{H}(\mathbf{u}) \neq 0$ we have $\delta_{j}(\mathbf{u}) \neq 0$ and $F_{\mathbf{u}, j}$ has non-zero discriminant. So $F_{\mathbf{u}, j}$ has the same number of zeros and degree as $F$, that is, the degree of $F_{\mathbf{u}, j}$ is $n \geq 2$ if $m \geq 3$ and $n \geq 3$ if $m=2$. Hence Propositions 6.4 and 6.5 are applicable. 
By precisely the same argument as in the case for Thue equations, there are $\mathbf{u} \in \mathbb{Z}^{q}$ and $j \in\{1, \ldots, D\}$ with (6.8) and (6.9). We proceed further with this $\mathbf{u}, j$.

We estimate the parameters corresponding to those in the bounds from Propositions 6.4, 6.5. First, we get precisely the same estimates as in (6.12) and (6.13). These imply

$$
\widehat{h} \leq(n+1) h\left(F_{\mathbf{u}, j}\right)+h\left(\delta^{(j)}(\mathbf{u})\right) \leq(n d)^{\exp O(r)}(h+1) .
$$

Further we have, similarly to (6.15),

$$
\left|\Delta_{K_{\mathbf{u}, j}}\right| \leq \exp \left\{(n d)^{\exp O(r)}(h+1)\right\}
$$

Next, similar to (6.17),

$$
|f(\mathbf{u})| \leq \exp \left\{(n d)^{\exp O(r)}(h+1)\right\} .
$$

The set $S$ now consists of places of $K_{\mathbf{u}, j}$ instead of the splitting field of $F_{\mathbf{u}, j}$ over $K$. So since $\left[K_{\mathbf{u}, j}: \mathbb{Q}\right] \leq D$ we now have $s \leq D(1+\omega)$, where $\omega$ is the number of distinct prime divisors of $f(\mathbf{u})$. This gives, instead of (6.18),

$$
s=O\left(D \log ^{*}|f(\mathbf{u})| / \log ^{*} \log ^{*}|f(\mathbf{u})|\right) .
$$

By inserting (6.32), and $D \leq d^{r}$, we obtain for the quantities $c_{3}, c_{4}$ in Propositions 6.4 and 6.5 the upper bounds

$$
c_{3}, c_{4} \leq \exp \left\{(n d)^{\exp O(r)}(h+1)\right\} .
$$

Lastly, we have instead of (6.20),

$$
P \leq Q \leq|f(\mathbf{u})|^{D} \leq \exp \left\{(n d)^{\exp O(r)}(h+1)\right\},
$$

where we have used (6.32) and $D \leq d^{r}$.

We now apply Propositions 6.4 and 6.5 to (6.29). Note that we have to take $L=K_{\mathbf{u}, j}$; so $d_{L} \leq D \leq d^{r}$. By inserting this and (6.30), (6.31), (6.34), (6.35) into the upper bounds from these Propositions, we obtain

$$
h\left(x^{(j)}(\mathbf{u})\right), h\left(y^{(j)}(\mathbf{u})\right) \leq \exp \left\{m^{3}(n d)^{\exp O(r)}(h+1)\right\} .
$$

By inserting this into (6.9), we obtain (3.20) in the case $q>0$.

Now let $q=0$. For $\alpha \in K$, write $\alpha^{(j)}$ for the conjugate of $\alpha$ corresponding to $w^{(j)}$, and let $F^{(j)}$ be the polynomial obtained by taking the $j$-th conjugates of the coefficients of $F$. We simply have to follow the above arguments, replacing everywhere $q$ by $0, \max (1,|\mathbf{u}|)$ by $1, K_{\mathbf{u}, j}$ by $K^{(j)}=\mathbb{Q}\left(w^{(j)}\right), F_{\mathbf{u}, j}$ by $F^{(j)}, x^{(j)}(\mathbf{u}), y^{(j)}(\mathbf{u})$ by $x^{(j)}, y^{(j)}$, and $f(\mathbf{u})$ by $f \in \mathbb{Z}$. Instead of (6.9) we 
have to use (6.25). Thus, we obtain the same estimate as (6.36), but with $x^{(j)}, y^{(j)}$ instead of $x_{j}(\mathbf{u}), y_{j}(\mathbf{u})$. Via $(6.25)$ we obtain (3.20) in the case $q=0$. This completes our proof of Proposition 3.7.

Proof of Proposition 3.8. Assume for the moment $q>0$. Let $x \in B, y \in$ $B \cap \overline{\mathbb{Q}}, m \in \mathbb{Z}_{\geq 2}$ be a solution of (3.18), such that $y \neq 0$ and $y$ is not a root of unity. Choose again $\mathbf{u}, j$ with $(6.8),(6.9)$. Note that $y^{(j)}(\mathbf{u})$ is a conjugate of $y$ since $y \in \overline{\mathbb{Q}}$; hence it is not 0 or a root of unity.

We apply Proposition 6.6 to (6.29). By (6.32), (6.33), we have for the constant $c_{5}$ in Proposition 6.6, that

$$
c_{5} \leq \exp \left\{(n d)^{\exp O(r)}(h+1)\right\} .
$$

Further, we have the upper bounds (6.30) for $\widehat{h},(6.31)$ for $\left|\Delta_{K_{\mathbf{u}, j}}\right|$, and (6.35) for $P$. By inserting these estimates into the upper bound for $m$ from Proposition 6.6, we obtain $m \leq \exp \left\{(n d)^{\exp O(r)}(h+1)\right\}$. In the case $q=0$, we obtain the same estimate, by making the same modifications as in the proof of Proposition 3.7. This finishes our proof of Proposition 3.8.

\section{REFERENCES}

[1] M. Aschenbrenner, Ideal membership in polynomial rings over the integers, $J$. Amer. Math. Soc., 17 (2004), 407-442.

[2] A. BAKER, Contributions to the theory of Diophantine equations I. On the representation of integers by binary forms, Philos. Trans. Roy. Soc. London Ser. A, $\mathbf{2 6 3}$ (1967/68), 173-191.

[3] A. BAKER, Bounds for the solutions of the hyperelliptic equation, Proc. Cambridge Philos. Soc., 65 (1969), 439-444.

[4] A. BÉRczes, J.-H. Evertse and K. Győry, Effective results for hyper- and superelliptic equations over number fields, Publ. Math. Debrecen, accepted for publication.

[5] E. Bombieri and W. Gubler, Heights in Diophantine geometry, Cambridge University Press, Cambridge, 2006.

[6] B. BrindzA, On $S$-integral solutions of the equation $y^{m}=f(x)$, Acta Math. Hungar., 44 (1984), 133-139.

[7] B. BRINDZA, On the equation $f(x)=y^{m}$ over finitely generated domains, Acta Math. Hungar., 53 (1989), 377-383.

[8] J. CoAtes, An effective p-adic analogue of a theorem of Thue, Acta Arith., 15 (1968/69), 279-305.

[9] J.-H. Evertse and K. GyŐRY, Effective results for unit equations over finitely generated integral domains, Math. Proc. Camb. Phil. Soc., (2013), to appear. 
[10] E. Friedman, Analytic formulas for the regulator of a number field., Invent. Math., 98 (1989), 599-622.

[11] K. GYÖRY, Bounds for the solutions of norm form, discriminant form and index form equations in finitely generated integral domains, Acta Math. Hungar., 42 (1983), 4580 .

[12] K. GYŐRY, Effective finiteness theorems for polynomials with given discriminant and integral elements with given discriminant over finitely generated domains, $J$. Reine Angew. Math., 346 (1984), 54-100.

[13] K. GYöRY and K. YU, Bounds for the solutions of $S$-unit equations and decomposable form equations, Acta Arith., 123 (2006), 9-41.

[14] G. Hermann, Die Frage der endlich vielen Schritte in der Theorie der Polynomideale, Math. Ann., 95 (1926), 736-788.

[15] S. Louboutin, Explicit bounds for residues of Dedekind zeta functions, values of $L$-functions at $s=1$, and relative class numbers, J. Number Theorey, 85 (2000), 263-282.

[16] R. C. Mason, Diophantine equations over function fields, Cambridge University Press, 1984.

[17] A. Schinzel and R. Tijdeman, On the equation $y^{m}=P(x)$, Acta Arith., 31 (1976), 199-204.

[18] W. M. Schmidt, Thue's equation over function fields, J. Austral. Math. Soc. Ser. A., 25 (1978), 385-442.

[19] V. G. Sprindžuk and S. V. Kotov, An effective analysis of the Thue-Mahler equation in relative fields (Russian), Dokl. Akad. Nauk. BSSR, 17 (1973), 393-395, 477.

[20] H. M. Stark, Some effective cases of the Brauer-Siegel theorem, Invent. Math., 23 (1974), 135-152.

[21] L. A. TrelinA, $S$-integral solutions of Diophantine equations of hyperbolic type (in Russian), Dokl. Akad. Nauk. BSSR, 22 (1978), 881-884;955.

[22] J. VÉGső, On superelliptic equations, Publ. Math. Debrecen, 44 (1994), 183-187.

[23] M. WALdschmidt, Diophantine approximation on linear algebraic groups, SpringerVerlag, 2000. 
A. BÉRCZES

Institute of Mathematics, University of Debrecen

H-4010 Debrecen, P.O. Box 12, Hungary

E-mail address: berczesa@science.unideb.hu

J.-H. Evertse

Universiteit Leiden, Mathematisch Institudt, Postbus 9512, 2300 RA Leiden, The Netherlands

E-mail address: evertse@math.leidenuniv.nl

K. GYŐRY

Institute of Mathematics, University of Debrecen H-4010 Debrecen, P.O. Box 12, Hungary

E-mail address: gyory@science.unideb.hu 